\title{
Effect of Perchloryl Fluoride Additions on the Flame Speed of Methane
}

\author{
Carl Halpern
}

(June 7, 1961)

\begin{abstract}
The effects of addition of small quantities of perchloryl fluoride and of oxygen on the flame speed of methane-air mixtures have been determined and are compared with each other and with the effect of moderately preheating the burning mixture. Only small additions of perchloryl fluoride, up to 3 percent by volume, could be used because of the corrosiveness of its combustion products. Perchloryl fluoride is more effective than oxygen in increasing the flame speed of methane but less effective, in the amounts added, than preheating the burning mixture to $330^{\circ} \mathrm{F}$. Maximum flame speeds for given experimental conditions were found to vary linearly with increasing additions of either perchloryl fluoride or oxygen.
\end{abstract}

\section{Introduction}

The determination of the effect on flame speed of small additions of perchloryl fluoride was carried on concurrently with another task in which gross effects in the combustion of hydrocarbons with perchloryl fluoride addition in a large duct were measured. Results of experiments in the duct reflected the combined influences of high temperature, variation of pressures, turbulence, and fuel-oxidant mixture ratio. Since closer control of conditions is possible in a small burner, the effects of the parameters considered on the flame speed are more clearly identified, and thus the information obtained is more basic. Methane was chosen as the fuel because we had had much experience in the determination of flame speeds of methane-air mixtures. At the conclusion of the experiments with perchloryl fluoride, a series of experiments was run with similar quantities of oxygen added to methane-air mixtures to compare the effects of oxygen and perchloryl fluoride.

Perchloryl fluoride $\left(\mathrm{ClO}_{3} \mathrm{~F}\right)$ is a relatively new compound, discovered in 1952 [1]. ${ }^{1}$ It is stated [2] to be an inert gas at room temperature, stable and noncorrosive when dry; its freezing point is $-146^{\circ} \mathrm{C}$ and its boiling point is $-46.8^{\circ} \mathrm{C}$. In the presence of water, it is very corrosive to most metals. Its heat of formation is $-5.12 \mathrm{kcal} /$ mole [3]; thus it can be expected, under the proper conditions, to be very active chemically, with high heats of reaction.

Lodwig and Margrave [4], in a study of perchloryl fluoride flames, have reported a few determinations of the flame speeds of mixtures of 65-75 percent methane and 35-25 percent perchloryl fluoride, by volume. Their values for flame speeds of these

1 Figures in brackets indicate the literature references at the end of this paper. mixtures range from about 185 to $195 \mathrm{~cm} / \mathrm{sec}$ (centimeters per second) and average about 190 $\mathrm{cm} / \mathrm{sec}(6.2 \mathrm{fps})$ (feet per second). However, their method for determining flame speeds, which is based on the measurement of the area of the visible inner cone of a Bunsen flame, is not now considered to yield reliable values. The preferred method is considered to be that based on the measurement of the schlieren cone $[5,6]$.

\section{Apparatus and Procedure}

A description of the apparatus and the method used to measure flame speeds has been presented earlier [7]. Briefly, this apparatus comprises drying and metering systems for both air and fuel, a conditioning chamber for the mixture of fuel and oxidant, and a nozzle, the exit of which is the burner port. Means are provided to control the temperature of the combustible mixture issuing from the nozzle.

For the present experiments it was decided to prepare mixtures of air and the added oxidant (perchloryl fluoride or oxygen) of the desired strength and to meter this mixture, rather than to set up a third metering system. Some error in the measurement of the flow of these mixtures was inevitable, since the calibration of the sharp-edged orifice used to meter the gas depended on the density of the gas. However, in view of the small quantities of additive used ( 5 percent by volume maximum for perchloryl fluoride and 6 percent for oxygen), it was felt that the error so introduced would be tolerable. The mixtures of air and added oxidant were prepared in a $120 \mathrm{gal}$ (16.05 cu ft) galvanized steel tank. The tank was evacuated to a pressure of several microns, and the oxidant was admitted from its container; pressure of the oxidant was measured by a mercury 
manometer, read to 0.01 in. Air from a compressor was dried by passing first through a column of activated alumina and then through a cold trap immersed in a slush of dry ice in a mixture of equal parts by weight of carbon tetrachloride and chloroform. It was then slowly admitted to the tank. Water content was thus kept to 0.03 percent by volume. The final pressure in the mixing tank was generally about 150 psig and was read on a calibrated Bourdon gage to $0.1 \mathrm{lb}$.

Because the products of combustion of perchloryl fluoride include hydrogen chloride and hydrogen fluoride, the exhaust gas was drawn by a large capacity vacuum pump from the enclosure surrounding the burner nozzle, and then through a chamber packed with copper turnings before being exhausted into the air.

Mixtures of $0.5,1,2,3,4$, and 5 percent by volume of perchloryl fluoride in air were prepared. For each mixture, attempts were made to determine the variation of flame speed with mixture ratio, by weight, of methane to air plus added perchloryl fluoride, with the velocity of the gas at the port of the nozzle held constant. The mixture ratio ranged from 0.054 to 0.072 and the velocity of the gas mixture at the port of the nozzle ranged from 4 to 7 fps.

Burning mixtures prepared with methane and air containing 1 and 2 percent, respectively, by volume of perchloryl fluoride yielded stable flames over the entire range of mixture ratios and gas velocities used.

Table 1 lists the conditions at which blowoff and flashback were encountered with the other mixtures. Blowoff represents the lean limit of operation of the burner, at which flames cannot be maintained; flashback represents the rich limit of operation.

TABLE 1. Limits of operation of burner

\begin{tabular}{|c|c|c|c|}
\hline $\begin{array}{c}\text { Percent } \mathrm{ClO}_{3} \mathrm{~F} \\
\text { by volume } \\
\text { added to air }\end{array}$ & $\begin{array}{l}\text { Percent } \mathrm{O}_{2} \\
\text { by volume } \\
\text { added to air }\end{array}$ & $\frac{\text { wt methane }}{\text { wt air+oxidant }}$ & $\begin{array}{c}\text { Gas velncity } \\
\text { fps }\end{array}$ \\
\hline
\end{tabular}

\begin{tabular}{l|l|l|l}
\hline \multicolumn{3}{|c|}{ Blowoff } \\
\hline 0.5 & 0 & 0.054 & 6 \\
.5 & 0 & .056 & 6 \\
.5 & 0 & .054 & 7 \\
.5 & 0 & .056 & 7 \\
0 & .5 & .054 & 6 \\
0 & .5 & .054 & 7 \\
0 & .5 & .056 & 6 \\
0 & 1.0 & .054 & 7 \\
0 & 1.0 & .054 & 7 \\
0 & 2.0 & .054 & \\
\hline
\end{tabular}

\begin{tabular}{c|r|r|c}
\hline \multicolumn{3}{|c}{ Flashback } \\
\hline 3.0 & 0 & .066 & 4 \\
0 & 6.0 & .066 & 4 \\
\hline
\end{tabular}

Formation of a white deposit on the lip of the port of the nozzle during combustion of methane with airperchloryl fluoride mixtures interfered with the gas flow and resulted in unstable, misshapen flames. Hydrogen fluoride and bydrogen chloride in the mantle of hot gas surrounding the inner cone of the flame undoubtedly had reacted with the metal of the nozzle to form a mixture of chlorides and fluorides. This deposit of salts was soft and easily removed. Rate of deposition was slow with weak mixtures of perchloryl fluoride and air; with proper care, stable conical flames were maintained. Rate of deposition with 4 and 5 percent mixtures, however, was so fast that undistorted flames could not be maintained. Hence, flame speeds could not be determined.

In a zone beginning about 0.03 in from the lip of the port of the nozzle and extending for about 0.2 in, the metal was eaten away to a depth of about 0.02 in, leaving a thin rim of untouched metal surrounding the port of the nozzle. During the first experiments with the 3 percent mixture of perchloryl fluoride, this rim burned through at one spot, thus creating permanently distorted flames. Experimentation with perchloryl fluoride was discontinued and the nozzle was removed from the burner system for repairs. Of the six bronze screens used to smooth the gas flow in the conditioning chamber of the burner assembly, the two top ones were heavily corroded and had to be replaced. Since flashback of perchloryl fluoride flames had taken place several times, it was felt that the corrosion of the screens was a result of these occurrences. The other four screens were in good condition. The reflecting surface of the spherical mirror used to focus light on the schlieren stop was eaten away in spots; this mirror was also replaced. The sharp-edged orifice used to meter the mixtures of air and perchloryl fluoride was in good condition.

Mixtures of air and $0.5,1,2,3,4,5$, and 6 percent, by volume, of added oxygen were prepared. Variation of flame speed with mixture ratio, by weight, of methane to air plus added oxygen was determined for each mixture. Velocity of the effluent gas at the nozzle port was held constant. As in the case of perchloryl fluoride, mixture ratio varied from 0.054 to 0.072 , and gas velocity ranged from 4 to $7 \mathrm{fps}$.

Burning mixtures made from air containing 3, 4, and 5 percent by volume of added oxygen gave stable flames over the entire range of mixture ratios and gas velocities used. Table 1 lists the conditions at which blowoff and flashback occurred with mixtures of other strengths. As mentioned above, these represent the lean and rich limits of operation for the burner.

The temperature controlled nozzle was used throughout these experiments, but the control of temperature was not as good as had been in previous experiments. Because of the limited supply of oxidant mixture, the nozzle could not be brought to thermal equilibrium, so there was a change in temperature during an individual run. The largest. temperature change during a run of about three hours' duration was a rise of nine degrees Fahrenheit; generally the temperature change did not exceed five degrees. Since the fluid circulating in the nozzle was cooled by tap water, temperature of the nozzle and hence of the incoming combustible mixture depended on the temperature of the tap water; as the experiments reported here lasted over a year, there was considerable seasonal variation 
in the nozzle temperature. The winter temperature was about $80-85^{\circ} \mathrm{F}$, while the summer temperature was about $95-100{ }^{\circ} \mathrm{F}$. The variation of flame speed with mixture temperature for methane-air flames had been found to be $0.0048 \mathrm{ft} /{ }^{\circ} \mathrm{F}$ [7]. The effect of temperature on methane-air-perchloryl fluoride and methane-air-oxygen flames, especially at low concentration of additives, should be of similar magnitude. Over a temperature range of $15{ }^{\circ} \mathrm{F}$, this would amount to about $0.07 \mathrm{fps}$. For a flame speed of about $1.5 \mathrm{fps}$, poor temperature control could introduce a variation of about 7 percent between summer and winter, and about 2 percent in an individual determination when the change of the temperature of the nozzle and of the incoming combustible mixture is $5^{\circ} \mathrm{F}$.

\section{Results}

Results of the addition of perchloryl fluoride are shown in figures 1 and 2 . Figure 1 shows the variation of flame speed with mixture ratio, by weight, of methane to air plus perchloryl fluoride at gas velocities, measured at the port of the nozzle, of 4 , 5 , 6, and 7 fps, when $0.5,1,2$, and 3 percent by volume, respectively, of perchloryl fluoride was added to the combustion air. Results obtained with burning mixtures made from the 0.5 and 1 percent perchloryl fluoride-air mixtures show the flame speed increasing as the mixture becomes richer in fuel, rising to a maximum and then decreasing. With the 2 percent mixture, the flame speed increases as the burning mixture becomes richer in fuel, and a maximum value seems to occur at mixture ratio of 0.072 . Only one set of observations could be made with the 3 percent mixture; at a gas velocity of $6 \mathrm{fps}$, flame speed increased as the burning mixture became richer in fuel until flashback occurred at a mixture ratio of 0.066 . Maximum flame speeds and the experimental conditions at which they occurred are listed in table 2 .

TABLE 2.-Maximum flame speeds for perchloryl fluoride addition and the experimental conditions at which they were obtained

\begin{tabular}{|c|c|c|c|c|}
\hline $\begin{array}{c}\text { Flame speed } \\
\text { fps }\end{array}$ & $\frac{\text { wt methane }}{\text { wt air }+\mathrm{ClO}_{3} \mathrm{~F}}$ & $\begin{array}{c}\text { Percent } \mathrm{ClO}_{3} \mathrm{~F} \\
\text { by volume } \\
\text { added to air }\end{array}$ & $\begin{array}{l}\text { Gas velocity } \\
\text { fps }\end{array}$ & $\begin{array}{c}\text { Gas temp. } \\
{ }^{\circ} \mathrm{F}\end{array}$ \\
\hline $\begin{array}{l}\text { 1. } 449 \\
\text { 1. } 475 \\
\text { 1. } 480 \\
1.455\end{array}$ & $\begin{array}{r}0.068 \\
.066 \\
.063 \\
.066\end{array}$ & $\begin{array}{r}0.5 \\
.5 \\
.5 \\
.5\end{array}$ & $\begin{array}{l}4 \\
5 \\
6 \\
7\end{array}$ & $\begin{array}{l}87.8 \\
88.0 \\
88.1 \\
88.4\end{array}$ \\
\hline $\begin{array}{l}\text { 1. } 652 \\
\text { 1. } 611 \\
1.705 \\
1.690\end{array}$ & $\begin{array}{l}.070 \\
.066 \\
.070 \\
.072\end{array}$ & $\begin{array}{l}1.0 \\
1.0 \\
1.0 \\
1.0\end{array}$ & $\begin{array}{l}4 \\
5 \\
6 \\
7\end{array}$ & $\begin{array}{l}93.1 \\
90.6 \\
92.4 \\
94.4\end{array}$ \\
\hline $\begin{array}{l}\text { 1. } 970 \\
\text { 2. } 082 \\
\text { 1. } 770 \\
\text { 2. } 123\end{array}$ & $\begin{array}{l}.072 \\
.072 \\
.072 \\
.072\end{array}$ & $\begin{array}{l}2.0 \\
2.0 \\
2.0 \\
2.0\end{array}$ & $\begin{array}{l}4 \\
5 \\
6 \\
7\end{array}$ & $\begin{array}{l}85.7 \\
90.3 \\
89.7 \\
88.4\end{array}$ \\
\hline 2. 386 & $.064^{*}$ & 3. 0 & 6 & 82.0 \\
\hline
\end{tabular}

* Flashback occurred at 0.066 .

As may be noted from the data listed in table 2, the addition of perchloryl fluoride to the combustible mixture shifted the mixture ratio at which maximum
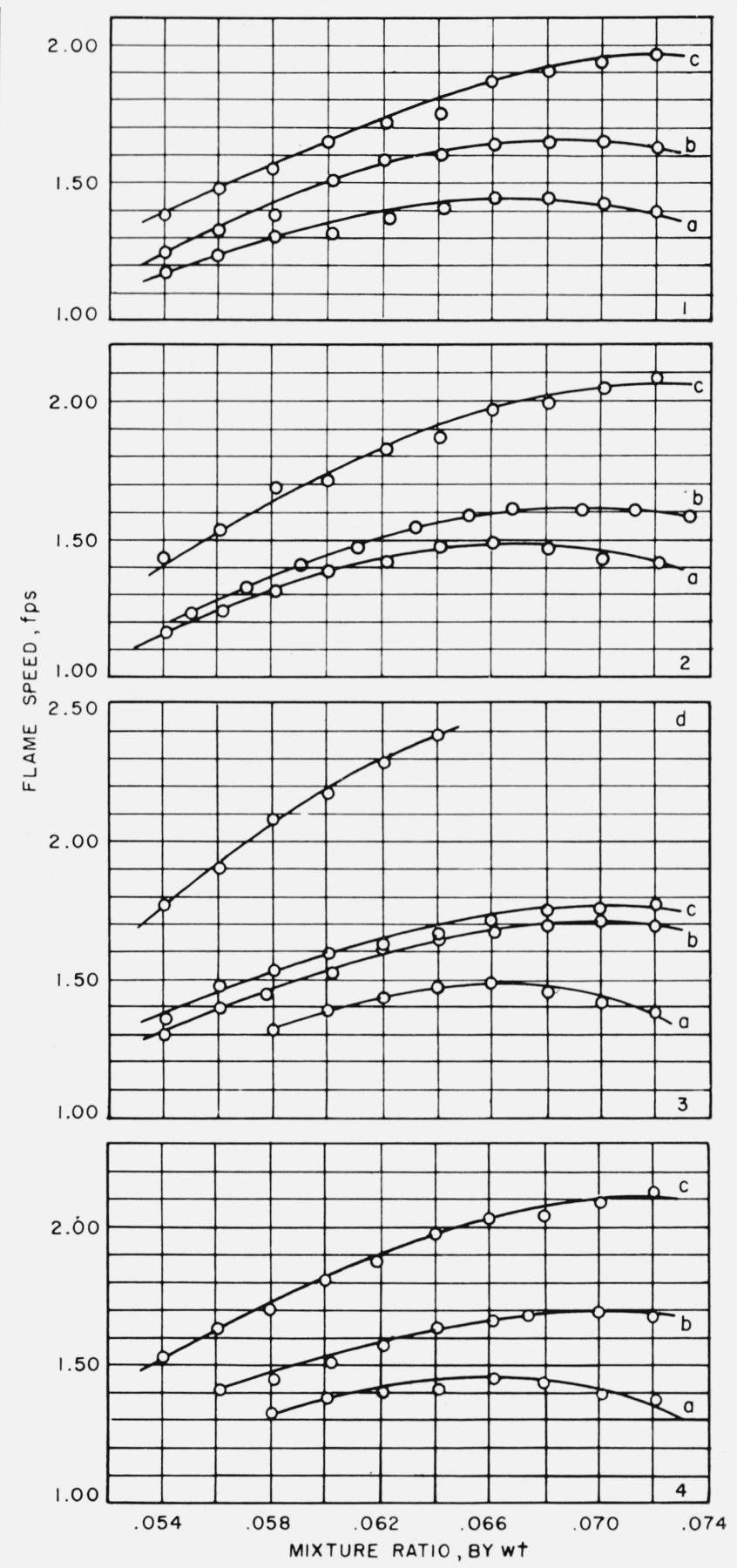

FIGURE 1. Effect of mixture ratio, by weight, of methane to air plus perchloryl fluoride on flame speed.

Percent, by volume, of added perchloryl fluoride $=a, 0.5 ; b, 1.0 ; c, 2.0$; and $d$, 3.0. Gas velocity $=1,4$ fps; 2,5 fps; 3,6 fps; and 4,7 fps.

flame speed occurs. It had been found previously [7] that maximum flame speed, $1.233 \mathrm{fps}$, for methane-air at a temperature of $84.4{ }^{\circ} \mathrm{F}$ occurred at mixture ratio of 0.062 . The addition of 2 percent by volume of perchloryl fluoride moved the maximum flame speed to a mixture ratio for methane to air plus perchloryl fluoride of 0.072 . 
Theoretically, flame speed is a property of the fuel and oxidant and of the physical condition of the mixture, and is independent of the apparatus in which it is measured. Hence, it was not expected that there be any variation of flame speed with gas velocity. However, there proved to be some variation. The 0.5 percent mixture of perchloryl fluoride in air showed practically no variation for lean burning mixtures. At richer mixtures, fuel-air plus perchloryl fluoride ratios of 0.068 and higher, there was
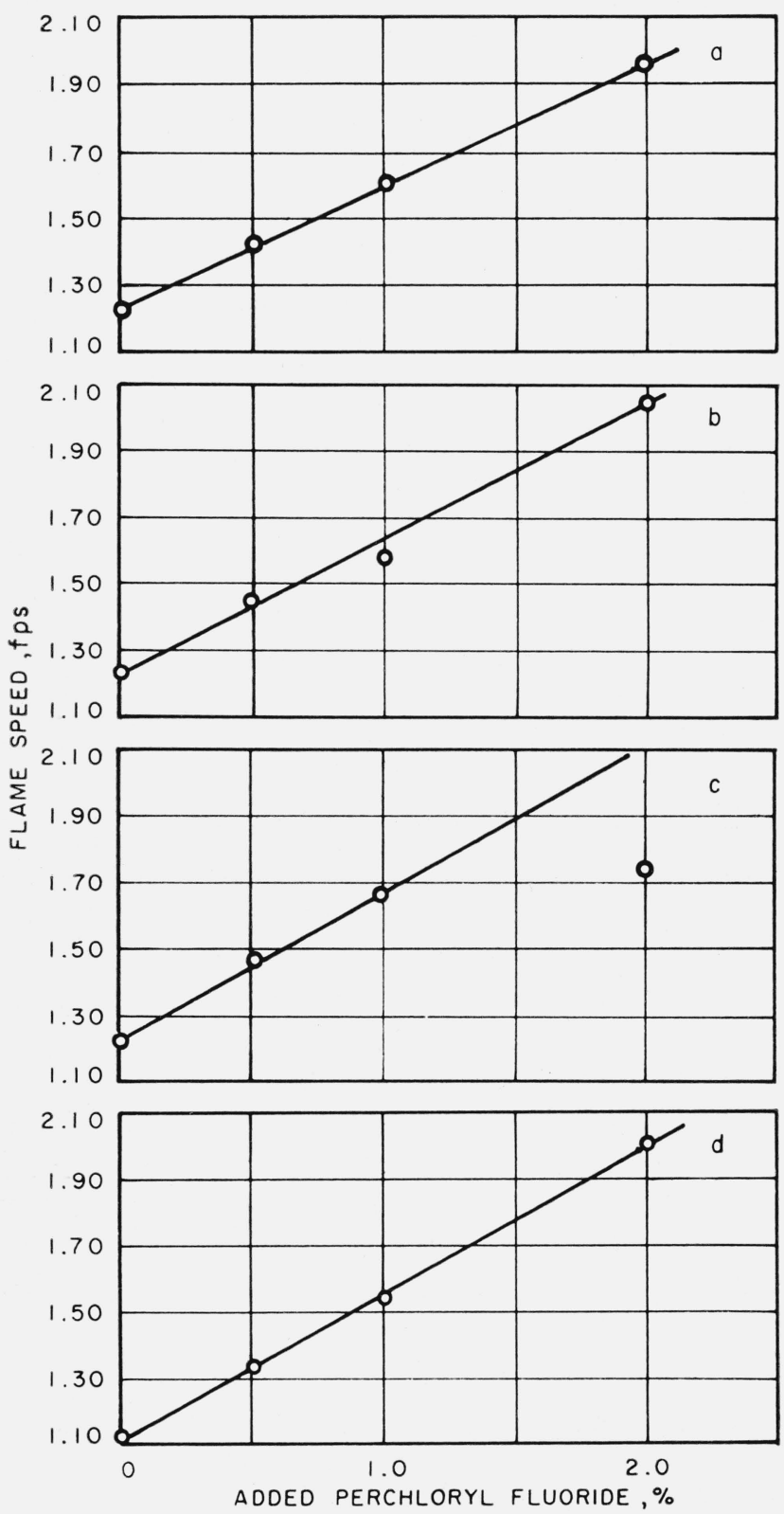

Frgure 2. Effect of added perchloryl fluoride on maximum flame speed.

Gas relocity=a, 4 fps; b, 5 fps; c, 6 fps; and d, 7 fps. a slight decrease of flame speed as gas velocity increases, which amounted to about 1.5 percent of the average value of the flame speed. The 1 percent mixture showed a small increase of flame speed as the gas velocity increases, also with a variation of about 1.5 percent. The 2 percent mixture showed a larger ncrease of flame speed with gas velocity, with a variation of 5 percent. (If the value of flame speed at $6 \mathrm{fps}$, which seems much too low, is ignored, then the variation becomes about 3 percent.) The flame speeds considered here have been corrected for temperature. The reasons for the variation of flame speed with gas velocity are obscure. Since these variations are small, they may be due to experimental difficulties, such as locating the flame front in the enlargements of the flame photographs, and thus they may fall within the experimental error. However, the trends seem to be consistent and, since a similar variation of flame speed with gas velocity had been found for methane-air flames previously [7], it is felt that the variation does exist. Conditions of the gas flow in the throat of the nozzle, e.g., the presence or absence, and the extent, of a boundary layer, are the most likely cause.

From the data which are listed in table 5, it may be shown that flame speed increases linearly with increasing percentage of perchloryl fluoride, at least over the small range of perchloryl fluoride addition covered here, and as the burning mixture becomes richer in fuel, the rate of increase of flame speed becomes larger. The values of flame speed at 0 percent perchloryl fluoride, that is, for a methane-air flame, are taken from our previous work [7]. In figure 2 is shown the relation of maximum flame speed for each gas velocity to the percentage of perchloryl fluoride added. Maximum flame speed varies linearly with the percentage of added perchloryl fluoride, and the rate of increase at each gas velocity is about the same.

Figures 3 and 4 show the effect of addition of oxygen on the flame speed of methane-air mixtures. Figure 3 shows the variation of flame speed with mixture ratio, by weight, of methane to air plus oxygen at constant gas velocity and at various strengths of added oxygen. The maximum flame speeds observed for each set of experimental conditions are listed in table 3 . It can be noted in figure 3 that, at low percentages of added oxygen, as the burning mixture becomes richer in fuel, the flame speed increases to a maximum and then decreases. As the burning mixture becomes richer in oxygen, the value of the mixture ratio at which the flame speed is a maximum gradually changes toward the fuel-rich side, and at 5 percent added oxygen, the maximum flame speed occurs about fuel to air plus oxygen ratio of 0.072 . With 6 percent added oxygen, themaximumflamespeed occurs beyond 0.072 ; i.e., an inflection point is not observed. 

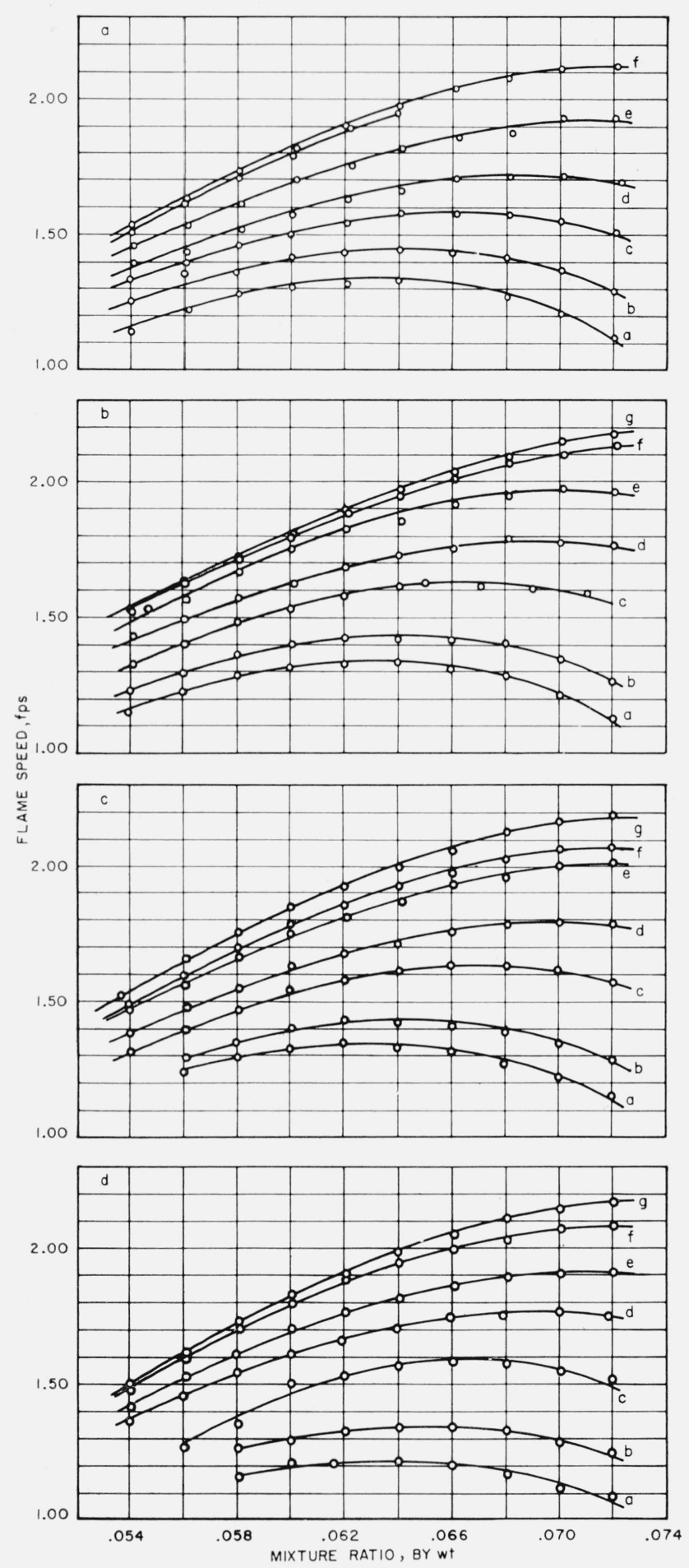

Figure 3(d). Effect of mixture ratio, by weight, of methane to air plus added oxygen on flame speed.

Percent added oxygen $=a, 0.5 ; \mathrm{b}, 1.0 ; \mathrm{c}, 2.0 ; \mathrm{d}, 3.0 ; \mathrm{e}, 4.0 ; \mathrm{f}, 5.0$; and $\mathrm{g}, 6.0$. Gas velocity $=\mathrm{A}, 4$ fps; B, 5 fps ' C, 6 fps; and $\mathrm{D}, 7$ fps.

Flame speeds of methane-air mixtures to which oxygen had been added also proved to vary somewhat with gas velocity. In general, the flame speed decreased as the gas velocity increased. However,

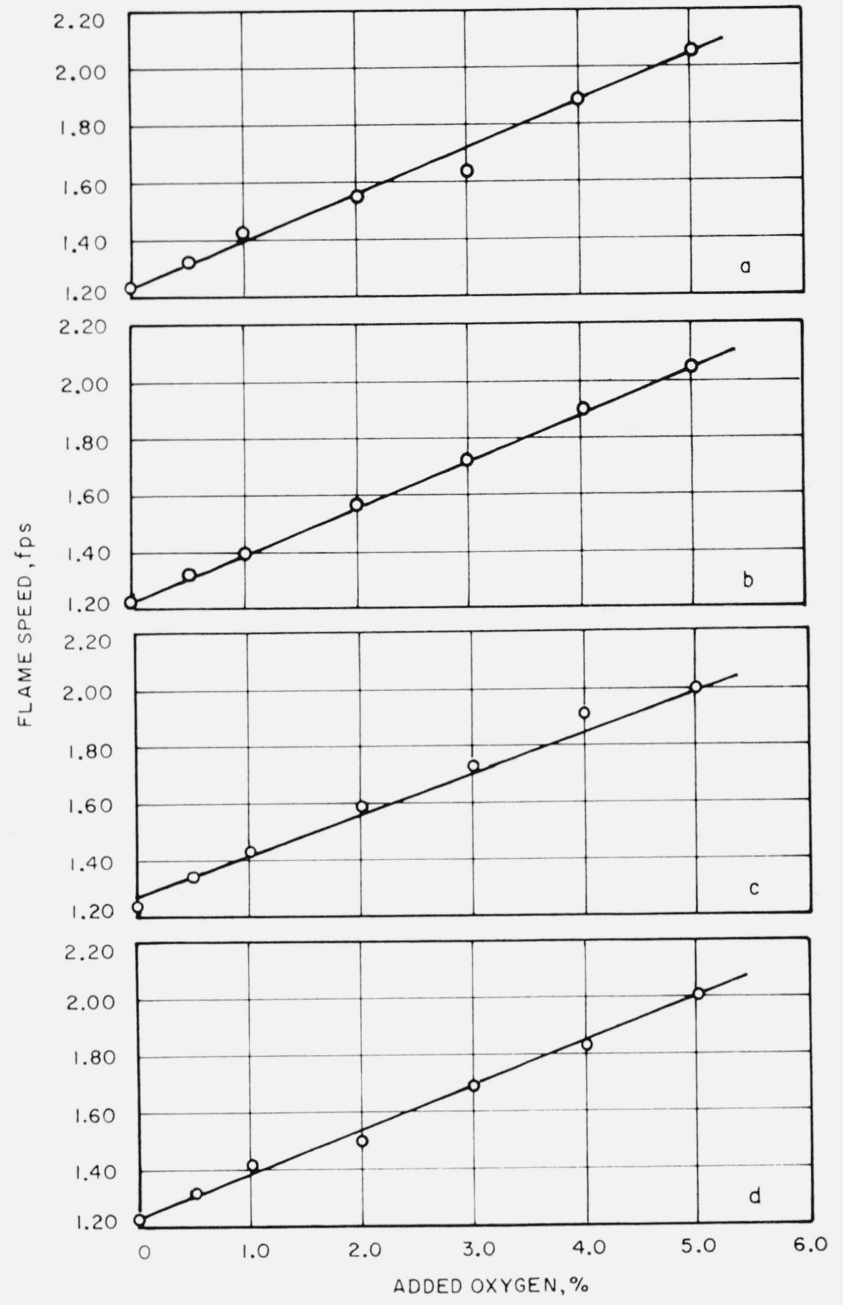

FIgurE 4. Effect of added oxygen on maximum flame speed. Gas velocity =a, 4 fps; b, 5 fps; c, 6 fps; and d, 7 fps.

there were a few instances where an increase of flame speed with increasing gas velocity occurred: at 0.5 percent oxygen addition, at mixture ratio of 0.072 ; at 1 percent oxygen addition, at mixture ratio of 0.072 ; and at 6 percent oxygen addition, at mixture ratios of $0.056,0.058,0.060,0.062,0.064,0.066$, and 0.068 . These variations of flame speeds, whether increase or decrease, are small and amount to about 1.2 percent of the average value of the flame speed. The flame speeds considered here have been corrected for temperature.

The data which are displayed in table 6 show the effect of increasing the amount of oxygen in the combustible mixture on the flame speed. It may be shown that flame speed increases linearly as the amount of oxygen is increased up to the addition of 4 or 5 percent; then the rate of increases changes abruptly and becomes less; the increase appears to be still linear. This effect is seen at all mixture ratios of fuel to air plus oxygen that were used and at all gas velocities. However, at gas velocities of 6 and $7 \mathrm{fps}$, the effect is less pronounced than at 4 and $5 \mathrm{fps}$. The rate of increase of flame speed (up 
to 4 or 5 percent oxygen added) is greater with mixtures that are fuel-rich. If, as in figure 4 , the maximum flame speed observed for each experimental condition is plotted against the percentage of oxygen added to the combustible mixture, then the result is a straight line and the slopes of the lines at each gas velocity are of similar magnitude. The values of flame speeds for 0 percent oxygen addition, that is, for methane-air, are taken from [7]. Since the maximum addition of oxygen was only 6 percent, one might question if there would not be a break in the curve at large oxygen addition. Lewis and von Elbe [8] reproduce a figure taken from the work of Jahn in which flame speed is plotted against the concentration of methane for "combustion airs" containing from 21 to 98.5 percent by volume of oxygen. A line drawn through the maximum flame speed found for each condition of "combustion air" is practically a straight line, showing a very slight degree of curvature, so that from 21 to 40 percent, by volume, of oxygen, the line is straight. In our experiments oxygen in the "combustion air" varied from 21 to 25.5 percent by volume.

TABLE 3. Maximum flame speeds for oxygen addition and the experimental conditions at which they were obtained

\begin{tabular}{|c|c|c|c|c|}
\hline $\begin{array}{l}\text { Flame speed } \\
\text { fps }\end{array}$ & $\frac{\text { wt methane }}{\text { wt air + oxygen }}$ & $\begin{array}{l}\text { Percent } \mathrm{O}_{2} \\
\text { by volume } \\
\text { added to air }\end{array}$ & $\begin{array}{c}\text { Gas velocity } \\
\text { fps }\end{array}$ & $\begin{array}{c}\text { Gas temp. } \\
{ }^{\circ} \mathrm{F}\end{array}$ \\
\hline 1. 322 & 0.064 & 0.5 & 4 & 849 \\
\hline 1.333 & .064 & .5 & 5 & 84. \\
\hline 1.343 & .062 & .5 & 6 & 87.2 \\
\hline 1. 318 & .064 & .5 & 7 & 86.9 \\
\hline 1.440 & .064 & 1.0 & 4 & 88.2 \\
\hline 1. 422 & .062 & 1. 0 & 5 & 88.6 \\
\hline 1. 436 & .062 & 1. 0 & 6 & 88.0 \\
\hline 1.443 & .066 & 1. 0 & 7 & 91.7 \\
\hline 1.583 & .064 & 2.0 & 4 & 92.4 \\
\hline 1. 634 & .065 & 2.0 & 5 & 95.7 \\
\hline 1. 634 & .068 & 2.0 & 6 & 97.4 \\
\hline 1. 581 & .066 & 2.0 & 7 & 95.2 \\
\hline 1.707 & .070 & 3.0 & 4 & 100.0 \\
\hline 1. 788 & .068 & 3. 0 & 5 & 97.0 \\
\hline 1.786 & .070 & 3. 0 & 6 & 98. \\
\hline 1. 765 & .070 & 3.0 & 7 & 101.3 \\
\hline 1.930 & .070 & 4.0 & 4 & 95.3 \\
\hline 1. 970 & .072 & 4. 0 & 5 & 98.4 \\
\hline 2.004 & .072 & 4. 0 & 6 & 102.4 \\
\hline 1.915 & .072 & 4. 0 & 7 & 102.4 \\
\hline 2.118 & .072 & 5. 0 & 4 & 98.3 \\
\hline 2.139 & .072 & 5.0 & 5 & 100.5 \\
\hline 2.067 & 072 & 5.0 & 6 & 101.2 \\
\hline 2. 080 & .072 & 5. 0 & 7 & 101.9 \\
\hline 1.943 & *. 064 & 6.0 & 4 & 91.2 \\
\hline 2. 169 & $>.072$ & 6.0 & 5 & 93.8 \\
\hline 2.187 & $>.072$ & 6. 0 & 6 & 95.8 \\
\hline 2.172 & $>.072$ & 6. 0 & 7 & 93.9 \\
\hline
\end{tabular}

*Flash-back occurred at 0.066 .

If it is assumed that the relation between maximum flame speed and oxygen content is, in fact, linear, then from the slope of the curves in figure 4 the maximum flame speed of methane in oxygen is estimated to be $16.5 \mathrm{fps}(503 \mathrm{~cm} / \mathrm{sec})$. For comparison with experimental values, Jahn [9] gives $330 \mathrm{~cm} / \mathrm{sec}$, Fiock [10] lists $393 \mathrm{~cm} / \mathrm{sec}$, Singer and Heimel [11] give 445 $\mathrm{cm} / \mathrm{sec}$, and Singer, Grumer, and Cook [12] give 375 and $440 \mathrm{~cm} / \mathrm{sec}$ for different types of burners. In a like manner from data given in figure 2 , it is possible to estimate the maximum flame speed for methane and perchloryl fluoride to be about $42 \mathrm{fps}(1,280$ $\mathrm{cm} / \mathrm{sec})$. However, since the extrapolation involved is quite large, and the assumed linearity of the relation of maximum flame speed with added perchoryl fluoride over the entire range up to 100 percent perchlorly fluoride quite uncertain, this value of 42 fps should be regarded with caution.

The addition of perchloryl fluoride is more effective than the addition of oxygen in increasing the flame speeds of methane-air mixtures. The greatest flame speed observed in these experiments was $2.386 \mathrm{fps}$; this occurred with the addition of 3 percent perchloryl fluoride by volume to the combustion air at a mixture ratio, by weight, of methane to air plus perchloryl fluoride of 0.064 . The gas velocity was 6 fps, the gas temperature, was $82.0^{\circ} \mathrm{F}$ and the water content was 0.03 percent by volume. The greatest flame speed observed on addition of oxygen was 2.187 fps; this was found with the addition of 6 percent oxygen by volume to the combustion air. Mixture ratio, by weight, of methane to air plus oxygen was 0.072 , gas velocity was $6 \mathrm{fps}$, gas temperature was $95.8^{\circ} \mathrm{F}$, and water content was 0.03 percent by volume. The above values for flame speeds should be compared with a maximum flame speed of 1.233 fps for a methane-air flame, mixture ratio, by weight, of methane to air of 0.062 , gas velocity of $6 \mathrm{fps}$, gas temperatuie of $84.4^{\circ} \mathrm{F}$, and water content, 0.03 percent by volume.

The heat of combustion of methane burning in perchloryl fluoride is calculated to be $-215.1 \mathrm{kcal} /$ mole, while the heat of combustion of methane in oxygen is only $-191.8 \mathrm{kcal} /$ mole. Since there is more energy available in the perchloryl fluoride reaction, it is to be expected that flame speeds should be larger.

The flame speed of methane-air mixtures had been found previously [7] to depend strongly on the initial temperature of the combustible mixture. Increasing the temperature increased the flame speed. At 330 ${ }^{\circ} \mathrm{F}$, the highest temperature used, maximum flame speed was 2.463 fps at a mixture ratio, by weight, of methane to air of 0.062 . Gas velocity was 5 fps at the port of the nozzle and the water content was 0.03 percent by volume. This value of flame speed should be compared to $2.386 \mathrm{fps}$, the greatest found for perchloryl fluoride addition, and $2.187 \mathrm{fps}$ for oxygen addition in the ranges covered in this report. From our previous work [7], it was found that to attain a flame speed of $2.386 \mathrm{fps}$ in a methane-air flame, it was necessary to preheat the mixture to about $320^{\circ} \mathrm{F}$ and to attain $2.187 \mathrm{fps}$, an initial gas temperature of about $295{ }^{\circ} \mathrm{F}$ is needed. Hence, it is apparent that a moderate increase in the initial temperature of the methane-air mixture is more effective in increasing flame speeds than is the addition of small quantities of other oxidants.

\section{Experimental Observations}

Tables 4,5 , and 6 present in detail some observations on the effect of some variables on the flame speed. 
TABLE 4. Effect of some variables on flame speed

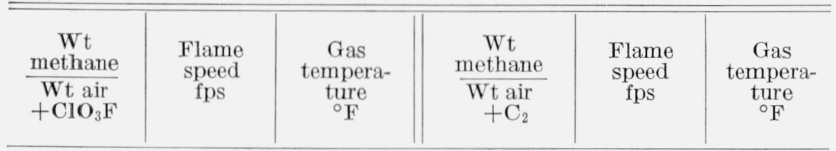

A. 0.5 percent by volume $\mathrm{ClO}_{3} \mathrm{~F}$ added to air

\begin{tabular}{|c|c|c|c|c|c|}
\hline \multicolumn{3}{|c|}{ 1. Gas velocity $=4 \mathrm{fps}$} & \multicolumn{3}{|c|}{ 2. Gas velocity $=5 \mathrm{fps}$} \\
\hline $\begin{array}{r}0.05404 \\
.05597 \\
.05805 \\
.06017 \\
.06217 \\
.06414 \\
.06603 \\
.06802 \\
.07006 \\
.07191\end{array}$ & $\begin{array}{l}1.172 \\
1.233 \\
1.303 \\
1.320 \\
1.376 \\
1.418 \\
1.445 \\
1.449 \\
1.421 \\
1.400\end{array}$ & $\begin{array}{l}85.2 \\
85.7 \\
86.1 \\
86.4 \\
86.8 \\
87.2 \\
87.4 \\
87.8 \\
88.0 \\
87.4\end{array}$ & $\begin{array}{r}0.05407 \\
.05661 \\
.05809 \\
.06003 \\
.06212 \\
.06406 \\
.06606 \\
.06806 \\
.07014 \\
.07212\end{array}$ & $\begin{array}{l}1.159 \\
1.241 \\
1.315 \\
1.385 \\
1.418 \\
1.470 \\
1.475 \\
1.462 \\
1.429 \\
1.412\end{array}$ & $\begin{array}{l}88.5 \\
85.2 \\
86.1 \\
86.7 \\
87.2 \\
87.8 \\
88.0 \\
88.4 \\
88.9 \\
88.9\end{array}$ \\
\hline \multicolumn{3}{|c|}{ 3. Gas velocity $=6 \mathrm{fps}$} & \multicolumn{3}{|c|}{ 4. Gas velocity $=7 \mathrm{fps}$} \\
\hline $\begin{array}{r}0.05802 \\
.06003 \\
.06206 \\
.06401 \\
.06601 \\
.06804 \\
.07002 \\
.07201\end{array}$ & $\begin{array}{l}1.315 \\
1.379 \\
1.430 \\
1.468 \\
1.480 \\
1.458 \\
1.414 \\
1.384\end{array}$ & $\begin{array}{l}88.6 \\
85.7 \\
86.7 \\
87.3 \\
88.1 \\
88.6 \\
89.2 \\
89.6\end{array}$ & $\begin{array}{r}0.05804 \\
.06002 \\
.06200 \\
.06404 \\
.06608 \\
.06797 \\
.07006 \\
.07201\end{array}$ & $\begin{array}{l}1.320 \\
1.379 \\
1.404 \\
1.406 \\
1.455 \\
1.434 \\
1.391 \\
1.370\end{array}$ & $\begin{array}{l}89.3 \\
89.7 \\
90.1 \\
88.3 \\
88.4 \\
89.0 \\
89.4 \\
89.9\end{array}$ \\
\hline & & & & & \\
\hline
\end{tabular}

B. 1 percent volume $\mathrm{ClO}_{3} \mathrm{~F}$ added to air

\begin{tabular}{|c|c|c|c|c|c|}
\hline \multicolumn{3}{|c|}{ 1. Gas velocity $=4 \mathrm{fps}$} & \multicolumn{3}{|c|}{ 2. Gas velocity $=5 \mathrm{fps}$} \\
\hline 0. 05404 & 1. 250 & 87.9 & 0.05496 & 1. 234 & 91.0 \\
\hline .05603 & $\begin{array}{l}\text { 1. } 328 \\
\text { lo }\end{array}$ & 88.6 & .05701 & $\begin{array}{l}\text { 1. } 321 \\
\text { 1. }\end{array}$ & 91.0 \\
\hline .05802 & 1. 380 & 89.2 & .05903 & 1. 406 & 90.6 \\
\hline 06012 & 1. 504 & 89.8 & .06108 & 1. 473 & 91.8 \\
\hline 06201 & 1. 578 & 90.3 & .06312 & 1. 546 & 89.9 \\
\hline .06410 & 1.600 & 90.7 & .06513 & 1. 580 & 90.6 \\
\hline 06603 & 1. 641 & 91.2 & .06669 & 1. 611 & 91.1 \\
\hline 06807 & $\begin{array}{l}1.646 \\
\text { 1. }\end{array}$ & 92.8 & .06926 & 1. 608 & 89.6 \\
\hline 07007 & $\begin{array}{l}1.652 \\
1.652\end{array}$ & 93.0 & .07128 & 1. 600 & 89.6 \\
\hline .07208 & 1. 637 & 93.1 & .07332 & 1. 587 & 91. 6 \\
\hline \multicolumn{3}{|c|}{ 3. Gas velocity $=6 \mathrm{fps}$} & \multicolumn{3}{|c|}{ 4. Gas velocity $=7 \mathrm{fps}$} \\
\hline 0. 05404 & 1. 306 & 91.1 & 0.05606 & 1. 403 & 95.1 \\
\hline .05605 & 1. 401 & 91.5 & .05815 & 1. 452 & 91.9 \\
\hline .05780 & 1. 457 & 91.4 & .06027 & 1. 510 & 91.4 \\
\hline .06015 & 1. 526 & 90.6 & .06208 & 1. 569 & 88.2 \\
\hline .06204 & 1. 627 & 93.4 & .06411 & 1. 625 & 90.0 \\
\hline .06402 & 1. 646 & 91. 5 & .06609 & 1. 664 & 91.2 \\
\hline .06606 & $\begin{array}{l}1.674 \\
1.670\end{array}$ & 90.8 & .06735 & $\begin{array}{l}\text { 1. } 676 \\
\text { lat }\end{array}$ & 92.7 \\
\hline .06802 & $\begin{array}{l}1.690 \\
1.690\end{array}$ & 91.8 & .07011 & $\begin{array}{l}1.090 \\
1.690\end{array}$ & 94.4 \\
\hline .07003 & 1. 705 & 92.4 & .07205 & 1. 669 & 95.6 \\
\hline .07204 & 1.690 & 93.2 & & & \\
\hline
\end{tabular}

C. 2 percent by volume $\mathrm{ClO}_{3} \mathrm{~F}$ added to air

\begin{tabular}{|c|c|c|c|c|c|}
\hline \multicolumn{3}{|c|}{ 1. Gas velocity $=4 \mathrm{fps}$} & \multicolumn{3}{|c|}{ 2. Gas velocity $=5 \mathrm{fps}$} \\
\hline 0.05403 & 1. 381 & 82.2 & 0.05402 & 1. 435 & 84.9 \\
\hline .05596 & 1.482 & 82.5 & .05601 & 1. 534 & 86.0 \\
\hline .05798 & 1. 544 & 83.1 & .05807 & 1. 681 & 86.5 \\
\hline .06003 & 1. 649 & 83.6 & .06004 & 1.712 & 86.6 \\
\hline .06197 & 1. 719 & 83.6 & .06207 & 1.828 & 87. 7 \\
\hline .06404 & 1.754 & 85.0 & .06407 & 1.869 & 88.4 \\
\hline 06597 & 1. 871 & 85.0 & .06604 & 1. 969 & 88.9 \\
\hline 06801 & 1. 903 & 85.6 & .06809 & 1. 987 & 89.0 \\
\hline .07000 & 1.945 & 85. 9 & .07009 & 2. 043 & 89.7 \\
\hline .07200 & 1. 970 & 85.7 & .07201 & 2. 082 & 90.3 \\
\hline \multicolumn{3}{|c|}{ 3. Gas velocity $=6 \mathrm{fps}$} & \multicolumn{3}{|c|}{ 4. Gas velocity $=7 \mathrm{fps}$} \\
\hline 0.05409 & 1. 357 & 87.9 & 0.05397 & 1.527 & 83.4 \\
\hline .05609 & 1. 474 & 88.4 & .05600 & 1. 632 & 84.2 \\
\hline .05806 & 1. 530 & 88.8 & .05799 & 1. 697 & 84.8 \\
\hline .06006 & 1.593 & 89.8 & .06000 & 1.800 & 85. 2 \\
\hline .06207 & 1. 609 & 84. 4 & .06187 & 1. 889 & 85.8 \\
\hline .06404 & 1. 660 & 86.4 & .06401 & 1.969 & 86. 0 \\
\hline .06597 & 1.710 & 87.8 & .06600 & 2. 027 & 86.5 \\
\hline .06801 & 1. 743 & 88.4 & .06796 & 2. 036 & 87. 2 \\
\hline .07002 & 1. 758 & 89.1 & .06999 & 2. 092 & 87. 9 \\
\hline .07206 & 1. 770 & 89.7 & .07199 & 2. 123 & 88.4 \\
\hline
\end{tabular}

TABLE 4. Effect of some variables on flame speed-Continued

\begin{tabular}{|c|c|c|c|c|c|}
\hline $\begin{array}{c}\text { Wt } \\
\text { methane } \\
\mathrm{Wt} \mathrm{air} \\
+\mathrm{ClO}_{3} \mathrm{~F}\end{array}$ & $\begin{array}{l}\text { Flame } \\
\text { speed } \\
\text { fps }\end{array}$ & $\begin{array}{l}\text { Gas } \\
\text { tempera- } \\
\text { ture } \\
{ }^{\circ} \mathrm{F}\end{array}$ & $\frac{\begin{array}{c}\mathrm{Wt} \\
\text { methane }\end{array}}{\begin{array}{c}\mathrm{Wt} \text { air } \\
+\mathrm{O}_{2}\end{array}}$ & $\begin{array}{l}\text { Flame } \\
\text { speed } \\
\text { fps }\end{array}$ & $\begin{array}{l}\text { Gas } \\
\text { tempera- } \\
\text { ture } \\
{ }^{\circ} \mathrm{F}\end{array}$ \\
\hline \multicolumn{3}{|c|}{$\begin{array}{l}\text { D. } 3 \text { percent by volume } \\
\mathrm{ClO}_{3} \mathrm{~F} \text { added to air }\end{array}$} & \multicolumn{3}{|c|}{$\begin{array}{l}\text { A. } 0.5 \text { percent by } \\
\text { volume } \mathrm{O}_{2} \text { added to air }\end{array}$} \\
\hline \multicolumn{3}{|c|}{ 1. Gas velocity $=6 \mathrm{fps}$} & \multicolumn{3}{|c|}{ 1. Gas velocity $=4 \mathrm{fps}$} \\
\hline $\begin{array}{r}0.05400 \\
.05601 \\
.05802 \\
.06003 \\
.06205 \\
.06400\end{array}$ & $\begin{array}{l}\text { 1. } 771 \\
\text { 1. } 904 \\
\text { 2. } 079 \\
\text { 2. } 168 \\
\text { 2. } 276 \\
\text { 2. } 386\end{array}$ & $\begin{array}{l}80.0 \\
79.8 \\
80.4 \\
80.3 \\
80.6 \\
82.0\end{array}$ & $\begin{array}{r}0.05411 \\
.05612 \\
.05807 \\
.06031 \\
.06210 \\
.06399 \\
.06807 \\
.07001 \\
.07210\end{array}$ & $\begin{array}{l}\text { 1. } 140 \\
\text { 1. } 223 \\
\text { 1. } 279 \\
\text { 1. } 305 \\
\text { 1. } 318 \\
\text { 1. } 322 \\
\text { 1. } 268 \\
\text { 1. } 209 \\
1.129\end{array}$ & $\begin{array}{l}80.4 \\
81.4 \\
82.4 \\
83.4 \\
84.3 \\
84.9 \\
86.0 \\
86.4 \\
86.8\end{array}$ \\
\hline
\end{tabular}

\begin{tabular}{|c|c|c|c|c|c|}
\hline$\frac{\begin{array}{c}\mathrm{Wt} \\
\text { methane }\end{array}}{\begin{array}{c}\mathrm{Wt} \text { air } \\
+\mathrm{O}_{2}\end{array}}$ & $\begin{array}{l}\text { Flame } \\
\text { speed } \\
\text { fps }\end{array}$ & $\begin{array}{c}\text { Gas } \\
\text { tempera- } \\
\text { ture } \\
\circ \mathrm{F}\end{array}$ & $\frac{\begin{array}{c}\mathrm{Wt} \\
\text { methane }\end{array}}{\begin{array}{c}\mathrm{Wt} \text { air } \\
+\mathrm{O}_{2}\end{array}}$ & $\begin{array}{l}\text { Flame } \\
\text { speed } \\
\text { fps }\end{array}$ & $\begin{array}{l}\text { Gas } \\
\text { tempera- } \\
\text { ture } \\
{ }^{\circ} \mathrm{F}\end{array}$ \\
\hline
\end{tabular}

A. 0.5 percent by volume $\mathrm{O}_{2}$ added to air

\begin{tabular}{|c|c|c|c|c|c|}
\hline \multicolumn{3}{|c|}{ 2. Gas velocity $=5 \mathrm{fps}$} & \multicolumn{3}{|c|}{ 3. Gas velocity $=6 \mathrm{fps}$} \\
\hline $\begin{array}{r}0.05398 \\
.05598 \\
.05802 \\
.05999 \\
.06201 \\
.06401 \\
.06598 \\
.06799 \\
.07003 \\
.07203\end{array}$ & $\begin{array}{l}\text { 1. } 147 \\
\text { 1. } 221 \\
\text { 1. } 280 \\
\text { 1. } 312 \\
\text { 1. } 327 \\
\text { 1. } 333 \\
\text { 1. } 308 \\
\text { 1. } 284 \\
\text { 1. } 215 \\
1.127\end{array}$ & $\begin{array}{l}81.3 \\
81.7 \\
82.5 \\
83.1 \\
83.8 \\
84.3 \\
85.1 \\
85.6 \\
86.1 \\
86.6\end{array}$ & $\begin{array}{r}0.05600 \\
.05803 \\
.06000 \\
.06200 \\
.06399 \\
.06600 \\
.06799 \\
.07001 \\
.07199\end{array}$ & $\begin{array}{l}1.240 \\
1.297 \\
1.326 \\
1.343 \\
1.329 \\
1.319 \\
1.268 \\
1.223 \\
1.158\end{array}$ & $\begin{array}{l}86.1 \\
86.6 \\
86.9 \\
87.2 \\
84.0 \\
84.8 \\
85.7 \\
86.4 \\
86.9\end{array}$ \\
\hline \multicolumn{3}{|c|}{$\begin{array}{l}\text { A. } 0.5 \text { percent by volume } \\
\mathrm{O}_{2} \text { addea to air }\end{array}$} & \multicolumn{3}{|c|}{$\begin{array}{l}\text { B. } 1.0 \text { percent by volume } \\
\mathrm{O}_{2} \text { added to air }\end{array}$} \\
\hline \multicolumn{3}{|c|}{ 4. Gas velocity $=7 \mathrm{fps}$} & \multicolumn{3}{|c|}{ 1. Gas velocity $=4 \mathrm{fps}$} \\
\hline $\begin{array}{r}0.05803 \\
.06005 \\
.06172 \\
.06400 \\
.06601 \\
06804 \\
.07001 \\
.07201\end{array}$ & $\begin{array}{l}\text { 1. } 258 \\
\text { 1. } 305 \\
\text { 1. } 305 \\
1.318 \\
\text { 1. } 301 \\
\text { 1. } 271 \\
\text { 1. } 219 \\
1.187\end{array}$ & $\begin{array}{l}89.7 \\
90.5 \\
86.3 \\
86.9 \\
87.6 \\
88.5 \\
89.4 \\
90.4\end{array}$ & $\begin{array}{r}0.05404 \\
.05599 \\
.05796 \\
.06004 \\
.06202 \\
.06406 \\
.06596 \\
.06801 \\
.07003 \\
.07206\end{array}$ & $\begin{array}{l}1.255 \\
\text { 1. } 358 \\
1.359 \\
1.418 \\
1.432 \\
1.440 \\
1.433 \\
1.416 \\
1.363 \\
1.298\end{array}$ & $\begin{array}{l}84.3 \\
85.3 \\
86.1 \\
86.7 \\
87.4 \\
88.2 \\
8.0 \\
89.2 \\
89.7 \\
90.1\end{array}$ \\
\hline
\end{tabular}

B. 1.0 percent by volume $\mathrm{O}_{2}$ added to air

\begin{tabular}{|c|c|c|c|c|c|}
\hline \multicolumn{3}{|c|}{ 2. Gas velocity $=5$ fps } & \multicolumn{3}{|c|}{ 3. Gas velocity $=6 \mathrm{fps}$} \\
\hline 0.05406 & 1. 224 & 86.0 & 0.05604 & 1. 293 & 90.6 \\
\hline .05598 & 1. 284 & 86.8 & .05799 & 1. 352 & 90.8 \\
\hline .05801 & 1. 359 & 87.4 & .06003 & 1. 404 & 91.3 \\
\hline .06003 & 1.400 & 88.0 & .06203 & 1. 436 & 88.0 \\
\hline .06201 & 1.422 & 88.6 & .06400 & 1. 426 & 88.8 \\
\hline .06402 & 1.418 & 89.4 & .06600 & 1.411 & 89.4 \\
\hline .06607 & 1. 417 & 89.9 & .06802 & 1. 384 & 90.1 \\
\hline .06803 & 1. 402 & 90.3 & .07003 & 1. 347 & 90.7 \\
\hline .07006 & 1. 342 & 90.6 & .07202 & 1. 290 & \\
\hline .07204 & 1.267 & 91.0 & & & \\
\hline \multicolumn{3}{|c|}{ B. 1.0 percent by volume $\mathrm{O}_{2}$ added to air } & \multicolumn{3}{|c|}{ C. 2.0 percent by volume $\mathrm{O}_{2}$ added to air } \\
\hline \multicolumn{3}{|c|}{ 4. Gas velocity $=7 \mathrm{fps}$} & \multicolumn{3}{|c|}{ 1. Gas velocity $=4 \mathrm{fps}$} \\
\hline 0.05802 & 1.365 & 93.6 & 0.05403 & 1.333 & 93.6 \\
\hline .05997 & 1.388 & 87.4 & .05607 & & 89.6 \\
\hline .06202 & 1.422 & 88. & .05807 & 1. 459 & 90.4 \\
\hline .06401 & 1.440 & 89.8 & .05999 & 1. 497 & 91.0 \\
\hline .06598 & 1.443 & 91.7 & .06207 & 1. 538 & 91.7 \\
\hline .06803 & 1.429 & 92.4 & .06404 & 1. 583 & 92.4 \\
\hline .07000 & 1. 389 & 92.8 & .06612 & 1. 579 & 92.8 \\
\hline .07196 & 1.351 & 93.7 & .06813 & 1. 571 & 93.3 \\
\hline & & & .07008 & $\begin{array}{l}1.543 \\
1.507\end{array}$ & $\begin{array}{l}93.7 \\
94.0\end{array}$ \\
\hline
\end{tabular}


TABLE 4. Effect of some variables on flame speed-Continued

\begin{tabular}{|c|c|c|c|c|c|}
\hline $\begin{array}{c}\mathrm{Wt} \\
\text { methane } \\
\mathrm{Wt} \text { air } \\
+\mathrm{O}_{2}\end{array}$ & $\begin{array}{l}\text { Flame } \\
\text { speed } \\
\text { fps }\end{array}$ & $\begin{array}{c}\text { Gas } \\
\text { tempera- } \\
\text { ture } \\
{ }^{\circ} \mathrm{F}\end{array}$ & $\begin{array}{c}\text { Wt } \\
\text { methane } \\
\begin{array}{c}\text { Wt air } \\
+\mathrm{O}_{2}\end{array}\end{array}$ & $\begin{array}{l}\text { Flame } \\
\text { speed } \\
\text { fps }\end{array}$ & $\begin{array}{c}\text { Gas } \\
\text { tempera- } \\
\text { tire } \\
{ }^{\circ} \mathrm{F}\end{array}$ \\
\hline \multicolumn{3}{|c|}{ 2. Gas velocity $=5 \mathrm{fps}$} & \multicolumn{3}{|c|}{ 3. Gas velocity $=6 \mathrm{fps}$} \\
\hline $\begin{array}{r}0.05412 \\
.05604 \\
.05804 \\
.06008 \\
.06206 \\
.06308 \\
.06502 \\
.06704 \\
.06898 \\
.07104\end{array}$ & $\begin{array}{l}1.334 \\
1.400 \\
1.480 \\
1.531 \\
1.579 \\
1.611 \\
1.634 \\
1.617 \\
1.607 \\
1.583\end{array}$ & $\begin{array}{l}97.0 \\
92.4 \\
93.4 \\
94.0 \\
94.5 \\
95.1 \\
95.7 \\
96.1 \\
96.7 \\
97.1\end{array}$ & $\begin{array}{r}0.05405 \\
.05609 \\
.05808 \\
.06004 \\
.06206 \\
.06407 \\
.06602 \\
.06804 \\
.06999 \\
.07205\end{array}$ & $\begin{array}{l}1.319 \\
1.397 \\
1.467 \\
1.540 \\
1.574 \\
1.614 \\
1.622 \\
1.634 \\
1.613 \\
1.576\end{array}$ & $\begin{array}{l}97.3 \\
93.2 \\
94.1 \\
94.7 \\
95.4 \\
96.3 \\
96.7 \\
97.4 \\
97.8 \\
98.4\end{array}$ \\
\hline \multicolumn{3}{|c|}{ C. 2.0 percent by volume $\mathrm{O}_{2}$ added to air } & \multicolumn{3}{|c|}{$\begin{array}{c}\text { D. } 3.0 \text { percent by volume of } \mathrm{O}_{2} \text { added to } \\
\text { air }\end{array}$} \\
\hline \multicolumn{3}{|c|}{ 4. Gas velocity $=7 \mathrm{fps}$} & \multicolumn{3}{|c|}{ 1. Gas velocity $=4 \mathrm{fps}$} \\
\hline $\begin{array}{r}0.05602 \\
.05802 \\
.06001 \\
.06203 \\
.06405 \\
.06602 \\
.06805 \\
.07001 \\
.07200\end{array}$ & $\begin{array}{l}1.371 \\
1.452 \\
1.506 \\
1.536 \\
1.574 \\
1.581 \\
1.579 \\
1.551 \\
1.523\end{array}$ & $\begin{array}{l}96.6 \\
95.3 \\
93.3 \\
93.9 \\
94.7 \\
95.2 \\
96.0 \\
96.5 \\
97.3\end{array}$ & $\begin{array}{r}0.05411 \\
.05606 \\
.05811 \\
.06004 \\
.06213 \\
.06409 \\
.06615 \\
.06815 \\
.07011 \\
.07226\end{array}$ & $\begin{array}{l}1.388 \\
1.433 \\
1.516 \\
1.571 \\
1.626 \\
1.659 \\
1.704 \\
1.707 \\
1.707 \\
1.696\end{array}$ & $\begin{array}{r}99.0 \\
94.2 \\
95.4 \\
96.4 \\
97.4 \\
98.1 \\
98.8 \\
99.4 \\
100.0 \\
100.5\end{array}$ \\
\hline \multicolumn{3}{|c|}{$\begin{array}{c}\text { D. } 3.0 \text { percent by volume of } \mathrm{O}_{2} \text { added to } \\
\text { air }\end{array}$} & \multirow{2}{*}{\multicolumn{3}{|c|}{ 3. Gas velocity $=6 \mathrm{fps}$}} \\
\hline \multicolumn{3}{|c|}{ 2. Gas velocity $=5 \mathrm{fps}$} & & & \\
\hline $\begin{array}{r}0.05413 \\
.05608 \\
.05809 \\
.06010 \\
.06210 \\
.06407 \\
.06610 \\
.06813 \\
.07011 \\
.07208\end{array}$ & $\begin{array}{l}1.429 \\
1.491 \\
1.563 \\
1.624 \\
1.679 \\
1.724 \\
1.758 \\
1.788 \\
1.776 \\
1.767\end{array}$ & $\begin{array}{l}97.7 \\
99.3 \\
94.4 \\
95.0 \\
95.4 \\
96.1 \\
96.7 \\
97.0 \\
97.7 \\
98.0\end{array}$ & $\begin{array}{r}0.05408 \\
.05610 \\
.05808 \\
.06006 \\
.06204 \\
.06404 \\
.06608 \\
.06806 \\
.07007 \\
.07204\end{array}$ & $\begin{array}{l}1.386 \\
1.479 \\
1.554 \\
1.627 \\
1.672 \\
1.714 \\
1.754 \\
1.784 \\
1.786 \\
1.781\end{array}$ & $\begin{array}{l}98.4 \\
93.7 \\
94.8 \\
95.5 \\
96.0 \\
96.8 \\
97.4 \\
98.0 \\
98.4 \\
98.7\end{array}$ \\
\hline D. 3.0 percen & y volume & added to air & \multicolumn{3}{|c|}{ E. 4.0 percent by volume $\mathrm{O}_{2}$ added to air } \\
\hline \multicolumn{3}{|c|}{ 4. Gas velocity $=7 \mathrm{fps}$} & \multicolumn{3}{|c|}{ 1. Gas velocity $=4 \mathrm{fps}$} \\
\hline $\begin{array}{r}0.05396 \\
.05594 \\
.05797 \\
.05992 \\
.06189 \\
.06391 \\
.06593 \\
.06793 \\
.06995 \\
.07188\end{array}$ & $\begin{array}{l}1.359 \\
1.454 \\
1,540 \\
1.612 \\
1.660 \\
1.704 \\
1.749 \\
1.756 \\
1.765 \\
1,756\end{array}$ & $\begin{array}{r}100.4 \\
100.7 \\
101.1 \\
101.9 \\
96.9 \\
98.2 \\
99.4 \\
100.5 \\
101.3 \\
102.3\end{array}$ & $\begin{array}{r}0.05415 \\
.05617 \\
.05816 \\
.06020 \\
.06222 \\
.06415 \\
.06624 \\
.06822 \\
.07018 \\
.07226\end{array}$ & $\begin{array}{l}1.459 \\
1.532 \\
1.612 \\
1.692 \\
1.752 \\
1.818 \\
1.851 \\
1.866 \\
1.930 \\
1.924\end{array}$ & $\begin{array}{l}91.0 \\
91.4 \\
92.0 \\
92.6 \\
93.2 \\
93.7 \\
94.2 \\
94.5 \\
94.9 \\
95.3\end{array}$ \\
\hline \multicolumn{3}{|c|}{ 2. Gas velocity $=5 \mathrm{fps}$} & \multicolumn{3}{|c|}{ 3. Gas velocity $=6 \mathrm{fps}$} \\
\hline 0.05467 & 1. 522 & 93.1 & 0.05405 & 1.476 & 95.4 \\
\hline .05609 & 1.571 & 93.8 & .05607 & 1. 559 & 96.3 \\
\hline .05806 & 1. 668 & 94.5 & .05810 & 1. 664 & 97.3 \\
\hline .06008 & 1. 746 & 95. 2 & .06004 & 1. 749 & 98.4 \\
\hline .06210 & 1.822 & $95 . \overline{7}$ & .06209 & 1.809 & 99.1 \\
\hline .06416 & 1. 853 & 96.6 & .06414 & 1.864 & 99.8 \\
\hline .06612 & 1.915 & 97.1 & .06607 & 1. 932 & 100.4 \\
\hline .06811 & 1. 950 & 97.8 & .06807 & 1. 959 & 101.1 \\
\hline .07012 & 1. 974 & 98.4 & .07006 & 1.996 & 101.8 \\
\hline .07210 & 1. 970 & 99.0 & .07202 & 2.004 & 102.4 \\
\hline
\end{tabular}

TABLE 4. Effect of some variables on flame speed-Continued

\begin{tabular}{|c|c|c|c|c|c|}
\hline $\begin{array}{c}\text { Wt } \\
\text { methane } \\
\begin{array}{c}\text { Wt air } \\
+\mathrm{O}_{2}\end{array}\end{array}$ & $\begin{array}{l}\text { Flame } \\
\text { speed } \\
\text { fps }\end{array}$ & $\begin{array}{c}\text { Gas } \\
\text { tempera- } \\
\text { ture } \\
{ }^{\circ} \mathrm{F}\end{array}$ & $\begin{array}{c}\mathrm{Wt} \\
\text { methane } \\
\begin{array}{c}\mathrm{Wt} \text { air } \\
+\mathrm{O}_{2}\end{array}\end{array}$ & $\begin{array}{l}\text { Flame } \\
\text { speed } \\
\text { fps }\end{array}$ & $\begin{array}{l}\text { Gas } \\
\text { tempera- } \\
\text { ture } \\
{ }^{\circ} \mathrm{F}\end{array}$ \\
\hline \multicolumn{3}{|c|}{$\begin{array}{l}\text { E. } 4.0 \text { percent by volume } \mathrm{O}_{2} \text { added } \\
\text { to air }\end{array}$} & \multicolumn{3}{|c|}{$\begin{array}{l}\text { F. } 5.0 \text { percent by volume } \mathrm{O}_{2} \text { added } \\
\text { to air }\end{array}$} \\
\hline \multicolumn{3}{|c|}{ 4. Gas velocity $=7 \mathrm{fps}$} & \multicolumn{3}{|c|}{ 1. Gas velocity $=4 \mathrm{fps}$} \\
\hline 0.05403 & 1.418 & 102. 2 & 0.05406 & 1. 536 & 93.2 \\
\hline & & & & & 94.3 \\
\hline & 1.6 & & & & 94.9 \\
\hline & 1.7 & & & & 95 \\
\hline & 1.7 & & & & \\
\hline & 1.8 & 101 & & & 96.7 \\
\hline .066 & 1. 872 & 102 & .066 & 2. 0 & 97.1 \\
\hline .06804 & 1.897 & 102 & .068 & & 97.6 \\
\hline .07003 & & & & & 97.8 \\
\hline .07197 & 1.915 & 102.0 & .07216 & 2.118 & 98.3 \\
\hline
\end{tabular}

F. 5.0 percent by volume $\mathrm{O}_{2}$ added to air

\begin{tabular}{|c|c|c|c|c|c|}
\hline \multicolumn{3}{|c|}{ 2. Gas velocity $=5 \mathrm{fps}$} & \multicolumn{3}{|c|}{ 3. Gas velocity $=6 \mathrm{fps}$} \\
\hline 0.05405 & 1.516 & 94. 3 & 0.05400 & 1. 487 & 94.0 \\
\hline .05606 & 1.624 & 95.3 & .05608 & 1. 593 & 95.3 \\
\hline .05805 & 1. 714 & 96. 2 & .05808 & 1. 697 & 96.5 \\
\hline .06008 & 1.794 & 97.0 & .06004 & 1.786 & 97.3 \\
\hline .06214 & 1.883 & 97.7 & .06203 & 1. 849 & 98.0 \\
\hline .06409 & 1. 949 & 98.1 & .06408 & $\begin{array}{l}1.926 \\
1.926\end{array}$ & 98.6 \\
\hline .06610 & 2. 004 & 98.7 & .06605 & $\begin{array}{l}\text { 1. } 970 \\
\text { 1. }\end{array}$ & 99.3 \\
\hline .06814 & 2. 068 & 99.4 & .06805 & 2. 025 & 100.0 \\
\hline .07013 & 2. 097 & $\begin{array}{l}35.7 \\
99.9\end{array}$ & .07006 & 2. 063 & 100.5 \\
\hline .07217 & 2. 139 & 100.5 & .07204 & 2. 067 & 101.2 \\
\hline
\end{tabular}

F. 5.0 percent by volume $\mathrm{O}_{2}$ added to air

\section{Gas velocity $=7 \mathrm{fps}$}

\begin{tabular}{r|r|r}
0.05400 & 1.478 & 92.8 \\
.05603 & 1.594 & 95.0 \\
.05802 & 1.708 & 96.4 \\
.06002 & 1.796 & 97.7 \\
.06202 & 1.887 & 98.6 \\
.06401 & 1.950 & 99.3 \\
.06602 & 1.996 & 99.9 \\
.06802 & 2.036 & 100.6 \\
.07005 & 2.068 & 101.3 \\
.07202 & 2.080 & 101.9
\end{tabular}

G. 6.0 percent by volume $\mathrm{O}_{2}$ added to air

\begin{tabular}{|c|c|c|c|c|c|}
\hline \multicolumn{3}{|c|}{ 2. Gas velocity $=5 \mathrm{fps}$} & \multicolumn{3}{|c|}{ 3. Gas velocity $=6 \mathrm{fps}$} \\
\hline 0.05404 & 1. 514 & 88.1 & 0.05366 & 1. 526 & 90.1 \\
\hline .05608 & 1. 620 & 89.0 & .05605 & 1. 660 & 90.5 \\
\hline .05802 & 1. 719 & 89.7 & .05808 & 1. 755 & 91.0 \\
\hline .06009 & 1. 800 & 90.3 & .06004 & 1. 846 & 91.8 \\
\hline .06205 & 1. 884 & 90.8 & .06208 & 1. 930 & 92.6 \\
\hline .06407 & 1. 963 & 91.5 & .06404 & 2. 001 & 93.1 \\
\hline .06607 & $\begin{array}{l}1.029 \\
2.029\end{array}$ & 92.1 & .06604 & 2. 062 & 94.0 \\
\hline .06812 & 2. 090 & 92.8 & .06806 & 2. 126 & 94.4 \\
\hline .07011 & 2.144 & 93.3 & .07006 & 2. 169 & 95.1 \\
\hline .07208 & 2. 169 & 93.8 & .07203 & 2.187 & 95.8 \\
\hline
\end{tabular}

4. Gas velocity $=7 \mathrm{fps}$

\begin{tabular}{l|l|l||l|l|l}
\hline 0.05403 & 1.494 & 88.0 & .06396 & 1.986 & 91.4 \\
.05605 & 1.610 & 88.7 & .06604 & 2.054 & 92.0 \\
.05807 & 1.734 & 89.5 & .06802 & 2.111 & 92.7 \\
.06001 & 1.833 & 90.2 & .07002 & 2.146 & 93.4 \\
.06202 & 1.904 & 90.9 & .07201 & 2.172 & 93.9
\end{tabular}


TABLE 5. Effect of perchloryl fluoride addition on flame speed of methane

\begin{tabular}{c|c||c|c}
$\begin{array}{c}\text { Vol. percent } \\
\mathrm{ClO}_{3} \mathrm{~F} \text { added } \\
\text { to air }\end{array}$ & Flame speed fps & $\begin{array}{c}\text { Vol. percent } \\
\mathrm{ClO}_{3} \mathrm{~F} \text { added } \\
\text { to air }\end{array}$ & Flame speed fps \\
&
\end{tabular}

A. Gas velocity $=4 \mathrm{fps}$

\begin{tabular}{|c|c|c|c|}
\hline \multicolumn{2}{|c|}{ 1. $\frac{\mathrm{Wt} \text { methane }}{\mathrm{Wt} \mathrm{air}+\mathrm{ClO}_{3} \mathrm{~F}}=0.054$} & \multicolumn{2}{|c|}{ 2. $\frac{\text { Wt methane }}{\mathrm{Wtair}+\mathrm{Cl} \mathrm{O}_{3} \mathrm{~F}}=0.056$} \\
\hline $\begin{array}{l}0.0 \\
0.5 \\
1.0 \\
2.0\end{array}$ & $\begin{array}{l}1.094 \\
1.168 \\
1.232 \\
1.392\end{array}$ & $\begin{array}{l}0.0 \\
0.5 \\
1.0 \\
2.0\end{array}$ & $\begin{array}{l}1.156 \\
1.226 \\
1.304 \\
1.492\end{array}$ \\
\hline \multicolumn{2}{|c|}{ 3. $\frac{\mathrm{Wt} \text { methane }}{\mathrm{Wt} \text { air }+\mathrm{Cl} \mathrm{O}_{3} \mathrm{~F}}=0.058$} & \multicolumn{2}{|c|}{ 4. $\frac{\text { Wt methane }}{\mathrm{Wt} \text { air }+\mathrm{ClO}_{3} \mathrm{~F}}=0.060$} \\
\hline $\begin{array}{l}0.0 \\
0.5 \\
\text { 1. } 0 \\
\text { 2. } 0\end{array}$ & $\begin{array}{l}\text { 1. } 188 \\
\text { 1. } 294 \\
\text { 1. } 356 \\
1.548\end{array}$ & $\begin{array}{l}0.0 \\
0.5 \\
1.0 \\
2.0\end{array}$ & $\begin{array}{l}\text { 1. } 219 \\
\text { 1. } 310 \\
1.477 \\
1.653\end{array}$ \\
\hline \multicolumn{2}{|c|}{ 5. $\frac{\mathrm{Wt} \text { methane }}{\mathrm{Wt} \mathrm{air}+\mathrm{ClO}_{3} \mathrm{~F}}=0.062$} & \multicolumn{2}{|c|}{ 6. $\frac{\text { Wt methane }}{\mathrm{Wt} \mathrm{air}+\mathrm{ClO}_{3} \mathrm{~F}}=0.064$} \\
\hline $\begin{array}{l}0.0 \\
0.5 \\
\text { J. } 0 \\
\text { c. } 0\end{array}$ & $\begin{array}{l}\text { 1. } 233 \\
\text { 1. } 364 \\
\text { 1. } 548 \\
\text { 1. } 723\end{array}$ & $\begin{array}{l}0.0 \\
0.5 \\
1.0 \\
2.0\end{array}$ & $\begin{array}{l}1.223 \\
1.404 \\
1.568 \\
1.751\end{array}$ \\
\hline \multicolumn{2}{|c|}{ 7. $\frac{\mathrm{Wt} \text { methane }}{\mathrm{Wt} \mathrm{air}+\mathrm{ClO}_{3} \mathrm{~F}}=0.066$} & \multicolumn{2}{|c|}{ 8. $\frac{\text { Wt methane }}{\mathrm{Wt} \mathrm{air}+\mathrm{ClO}_{3} \mathrm{~F}}=0.068$} \\
\hline $\begin{array}{l}0.0 \\
0.5 \\
1.0 \\
\text { 2. } 0\end{array}$ & $\begin{array}{l}1.190 \\
1.433 \\
1.622 \\
1.868\end{array}$ & $\begin{array}{l}0.0 \\
0.5 \\
1.0 \\
2.0\end{array}$ & $\begin{array}{l}1.148 \\
\text { 1. } 432 \\
1.604 \\
1.897\end{array}$ \\
\hline \multicolumn{2}{|c|}{ 9. $\frac{\mathrm{Wt} \text { methane }}{\mathrm{Wt} \text { air }+\mathrm{ClO}_{3} \mathrm{~F}}=0.070$} & \multicolumn{2}{|c|}{ 10. $\frac{\text { Wt methane }}{\mathrm{Wt} \mathrm{air}+\mathrm{ClO}_{3} \mathrm{~F}}=0.072$} \\
\hline $\begin{array}{l}0.0 \\
0.5 \\
1.0 \\
\text { 2. } 0\end{array}$ & $\begin{array}{l}\text { 1. } 094 \\
\text { 1. } 403 \\
\text { 1. } 609 \\
\text { 1. } 937\end{array}$ & $\begin{array}{l}0.0 \\
0.5 \\
1.0 \\
2.0\end{array}$ & $\begin{array}{l}1.017 \\
1.385 \\
1.593 \\
1.963\end{array}$ \\
\hline
\end{tabular}

B. Gas velocity $=5 \mathrm{fps}$

\begin{tabular}{|c|c|c|c|}
\hline \multicolumn{2}{|c|}{ 1. $\frac{\mathrm{Wt} \text { methane }}{\mathrm{Wt} \text { air }+\mathrm{ClO}_{3} \mathrm{~F}}=0.054$} & \multicolumn{2}{|c|}{ 2. $\frac{\mathrm{Wt} \text { methane }}{\mathrm{Wt} \text { air }+\mathrm{ClO}_{3} \mathrm{~F}}=0.056$} \\
\hline $\begin{array}{l}0.0 \\
0.5 \\
1.0 \\
2.0\end{array}$ & $\begin{array}{l}1.094 \\
1.138 \\
1.201 \\
1.432\end{array}$ & $\begin{array}{l}0.0 \\
0.5 \\
1.0 \\
2.0\end{array}$ & $\begin{array}{l}1.156 \\
1.237 \\
1.288 \\
1.526\end{array}$ \\
\hline \multicolumn{2}{|c|}{ 3. $\frac{\mathrm{Wt} \text { methane }}{\mathrm{Wt} \mathrm{air}+\mathrm{ClO}_{3} \mathrm{~F}}=0.058$} & \multicolumn{2}{|c|}{ 4. $\frac{\mathrm{Wt} \text { methane }}{\mathrm{Wt} \text { air }+\mathrm{ClO}_{3} \mathrm{~F}}=0.060$} \\
\hline $\begin{array}{l}0.0 \\
0.5 \\
1.0 \\
2.0\end{array}$ & $\begin{array}{l}1.188 \\
1.306 \\
1.375 \\
1.436\end{array}$ & $\begin{array}{l}0.0 \\
0.5 \\
1.0 \\
2.0\end{array}$ & $\begin{array}{l}\text { 1. } 219 \\
\text { 1. } 372 \\
1.436 \\
1.670\end{array}$ \\
\hline \multicolumn{2}{|c|}{ 5. $\frac{\mathrm{Wt} \text { methane }}{\mathrm{Wt} \text { air }+\mathrm{ClO}_{3} \mathrm{~F}}=0.062$} & \multicolumn{2}{|c|}{ 6. $\frac{\mathrm{Wt} \text { methane }}{\mathrm{Wt} \text { air }+\mathrm{C} 1 \mathrm{O}_{3} \mathrm{~F}}=0.064$} \\
\hline $\begin{array}{l}0.0 \\
0.5 \\
1.0 \\
2.0\end{array}$ & $\begin{array}{l}1.233 \\
1.404 \\
1.518 \\
1.701\end{array}$ & $\begin{array}{l}0.0 \\
0.5 \\
1.0 \\
2.0\end{array}$ & $\begin{array}{l}1.223 \\
1.453 \\
1.549 \\
1.849\end{array}$ \\
\hline
\end{tabular}

TABLE 5. Effect of perchloryl fluoride addition on flame speed of methane -Continued

\begin{tabular}{|c|c|c|c|}
\hline $\begin{array}{l}\text { Vol. percent } \\
\mathrm{ClO}_{3} \mathrm{~F} \text { added } \\
\text { to air }\end{array}$ & Flame speed fps & $\begin{array}{l}\text { Vol. percent } \\
\mathrm{ClO}_{3} \mathrm{~F} \text { added } \\
\text { to air }\end{array}$ & Flame speed fps \\
\hline \multicolumn{2}{|c|}{$7 . \frac{\mathrm{Wt} \text { methane }}{\mathrm{Wt} \text { air }+\mathrm{ClO}_{3} \mathrm{~F}}=0.066$} & \multicolumn{2}{|c|}{ 8. $\frac{\mathrm{Wt} \text { methane }}{\mathrm{Wt} \text { air }+\mathrm{ClO}_{3} \mathrm{~F}}=0.068$} \\
\hline $\begin{array}{l}0.0 \\
0.5 \\
1.0 \\
2.0\end{array}$ & $\begin{array}{l}1.190 \\
1.457 \\
1.577 \\
1.946\end{array}$ & $\begin{array}{l}0.0 \\
0.5 \\
1.0 \\
2.0\end{array}$ & $\begin{array}{l}1.148 \\
1.442 \\
1.582 \\
1.964\end{array}$ \\
\hline \multicolumn{2}{|c|}{ 9. $\frac{\mathrm{Wt} \text { methane }}{\mathrm{Wt} \text { air }+\mathrm{ClO}_{3} \mathrm{~F}}=0.070$} & \multicolumn{2}{|c|}{ 10. $\frac{\mathrm{Wt} \text { methane }}{\mathrm{Wt} \text { air }+\mathrm{ClO}_{3} \mathrm{~F}}=0.072$} \\
\hline $\begin{array}{l}0.0 \\
0.5 \\
1.0 \\
2.0\end{array}$ & $\begin{array}{l}1.094 \\
1.406 \\
1.574 \\
2.016\end{array}$ & $\begin{array}{l}0.0 \\
0.5 \\
1.0 \\
2.0\end{array}$ & $\begin{array}{l}1.017 \\
1.389 \\
1.551 \\
2.052\end{array}$ \\
\hline
\end{tabular}

C. Gas velocity $=6 \mathrm{fps}$

\begin{tabular}{|c|c|c|c|}
\hline \multicolumn{2}{|c|}{ 1. $\frac{\mathrm{Wt} \text { methane }}{\mathrm{Wt} \text { air }+\mathrm{ClO}_{3} \mathrm{~F}}=0.054$} & \multicolumn{2}{|c|}{ 2. $\frac{\mathrm{Wt} \text { methane }}{\mathrm{Wt} \text { air }+\mathrm{ClO}_{3} \mathrm{~F}}=0.056$} \\
\hline 0.0 & 1. 094 & 0.0 & 1. 156 \\
\hline $\begin{array}{l}0.5 \\
1.0\end{array}$ & 1.272 & $\begin{array}{l}0.5 \\
1.0\end{array}$ & 1.365 \\
\hline 2.0 & 1. 339 & 2. 0 & 1. 452 \\
\hline 3. 0 & 1. 749 & 3. 0 & 1. 881 \\
\hline \multicolumn{2}{|c|}{ 3. $\frac{\text { Wt methane }}{\text { Wt air }+\mathrm{ClO}_{3} \mathrm{~F}}=0.058$} & \multicolumn{2}{|c|}{ 4. $\frac{\text { Wt methane }}{\mathrm{Wt} \mathrm{air}+\mathrm{ClO}_{3} \mathrm{~F}}=0.060$} \\
\hline 0.0 & 1.188 & 0.0 & 1. 219 \\
\hline 0.5 & 1. 304 & 0.5 & 1. 372 \\
\hline 1.0 & 1. 422 & 1.0 & 1. 495 \\
\hline 2. 0 & 1.508 & 2.0 & 1. 566 \\
\hline 3.0 & 2. 059 & 3.0 & 2.147 \\
\hline \multicolumn{2}{|c|}{ 5. $\frac{\text { Wt methane }}{\mathrm{Wt} \text { air }+\mathrm{ClO}_{3} \mathrm{~F}}=0.062$} & \multicolumn{2}{|c|}{ 6. $\frac{\text { Wt methane }}{\text { Wt air }+\mathrm{ClO}_{3} \mathrm{~F}}=0.064$} \\
\hline 0.0 & 1. 233 & 0.0 & 1. 223 \\
\hline 0.5 & 1. 418 & 0.5 & 1. 453 \\
\hline 1. 0 & 1. 582 & 1.0 & 1.610 \\
\hline 3. 0 & $\begin{array}{l}1.089 \\
2.257\end{array}$ & 3. 0 & 2. 374 \\
\hline \multicolumn{2}{|c|}{ 7. $\frac{\text { Wt methane }}{\mathrm{Wt} \text { air }+\mathrm{ClO}_{3} \mathrm{~F}}=0.066$} & \multicolumn{2}{|c|}{ 8. $\frac{\text { Wt methane }}{\text { Wt air }+\mathrm{ClO}_{3} \mathrm{~F}}=0.068$} \\
\hline 0.0 & 1.190 & 0.0 & 1.148 \\
\hline 0.5 & 1.461 & 0.5 & 1.437 \\
\hline 1. 0 & 1. 632 & 1.0 & 1.653 \\
\hline 2.0 & 1.693 & 2.0 & 1. 723 \\
\hline \multicolumn{2}{|c|}{ 9. $\frac{\mathrm{Wt} \text { methane }}{\mathrm{Wt} \text { air }+\mathrm{ClO}_{3} \mathrm{~F}}=0.070$} & \multicolumn{2}{|c|}{ 10. $\frac{\text { Wt methane }}{\text { Wt air }+\mathrm{ClO}_{3} \mathrm{~F}}=0.072$} \\
\hline 0.0 & 1. 094 & 0.0 & 1. 017 \\
\hline 0.5 & 1. 390 & 0.5 & 1. 358 \\
\hline 1.0 & 1. 665 & 1.0 & 1. 646 \\
\hline 2.0 & 1. 734 & 2.0 & 1. 743 \\
\hline
\end{tabular}

D. Gas velocity $=7 \mathrm{fps}$

\begin{tabular}{c|c|c|c}
\hline 1. $\frac{\text { Wt methane }}{\mathrm{Wt} \text { air }+\mathrm{ClO}_{3} \mathrm{~F}}=0.054$ & \multicolumn{2}{c}{ 2. $\frac{\text { Wt methane }}{\mathrm{Wt} \text { air }+\mathrm{ClO}_{3} \mathrm{~F}}=0.056$} \\
\hline 0.0 & 1.094 & 0.0 & 1.156 \\
0.5 & -1.5 & 0.5 & 1.349 \\
1.0 & 1.532 & 2.0 & 1.633
\end{tabular}


TABLE 5. Effect of perchloryl fluoride addition on flame speed of methane-Continued

\begin{tabular}{|c|c|c|c|}
\hline $\begin{array}{l}\text { Vol. percent } \\
\mathrm{ClO}_{3} \mathrm{~F} \text { added } \\
\text { to air }\end{array}$ & Flame speed fps & $\begin{array}{c}\text { Vol. percent } \\
\mathrm{ClO}_{3} \mathrm{~F} \text { added } \\
\text { to air }\end{array}$ & Flame speed fps \\
\hline \multicolumn{2}{|c|}{ 3. $\frac{\mathrm{Wt} \text { methane }}{\mathrm{Wt} \text { air }+\mathrm{ClO}_{3} \mathrm{~F}}=0.058$} & \multicolumn{2}{|c|}{ 4. $\frac{\text { Wt methane }}{\mathrm{Wt} \text { air }+\mathrm{ClO}_{3} \mathrm{E}}=0.060$} \\
\hline $\begin{array}{l}0.0 \\
0.5 \\
1.0 \\
2.0\end{array}$ & $\begin{array}{l}1.188 \\
1.295 \\
1.414 \\
1.695\end{array}$ & $\begin{array}{l}0.0 \\
0.5 \\
1.0 \\
2.0\end{array}$ & $\begin{array}{l}1.219 \\
1.352 \\
1.475 \\
1.760\end{array}$ \\
\hline \multicolumn{2}{|c|}{ 5. $\frac{\text { Wt methane }}{\mathrm{Wt} \text { air }+\mathrm{ClO}_{3} \mathrm{~F}}=0.062$} & \multicolumn{2}{|c|}{ 6. $\frac{\text { Wt methane }}{\mathrm{Wt} \text { air }+\mathrm{ClO}_{3} \mathrm{~F}}=0.064$} \\
\hline $\begin{array}{l}0.0 \\
0.5 \\
1.0 \\
2.0\end{array}$ & $\begin{array}{l}1.233 \\
1.375 \\
1.550 \\
1.882\end{array}$ & $\begin{array}{l}0.0 \\
0.5 \\
1.0 \\
2.0\end{array}$ & $\begin{array}{l}1.223 \\
1.386 \\
1.597 \\
1.961\end{array}$ \\
\hline \multicolumn{2}{|c|}{ 7. $\frac{\text { Wt methane }}{\mathrm{Wt} \text { air }+\mathrm{ClO}_{3} \mathrm{~F}}=0.066$} & \multicolumn{2}{|c|}{ 8. $\frac{\text { Wt methane }}{\mathrm{Wt} \text { air }+\mathrm{ClO}_{3} \mathrm{~F}}=0.068$} \\
\hline $\begin{array}{l}0.0 \\
0.5 \\
1.0 \\
2.0\end{array}$ & $\begin{array}{l}1.190 \\
1.435 \\
1.630 \\
2.016\end{array}$ & $\begin{array}{l}0.0 \\
0.5 \\
1.0 \\
2.0\end{array}$ & $\begin{array}{l}1.148 \\
1.411 \\
1.634 \\
2.022\end{array}$ \\
\hline \multicolumn{2}{|c|}{ 9. $\frac{\text { Wt methane }}{\mathrm{Wt} \text { air }+\mathrm{ClO}_{3} \mathrm{~F}}=0.070$} & \multicolumn{2}{|c|}{ 10. $\frac{\text { Wt methane }}{\text { Wt air }+\mathrm{ClO}_{3} \mathrm{~F}}=0.072$} \\
\hline $\begin{array}{l}0.0 \\
0.5 \\
1.0 \\
2.0\end{array}$ & $\begin{array}{l}1.094 \\
1.366 \\
1.640 \\
2.074\end{array}$ & $\begin{array}{l}0.0 \\
0.5 \\
1.0 \\
2.0\end{array}$ & $\begin{array}{l}1.017 \\
1.342 \\
1.613 \\
2.103\end{array}$ \\
\hline
\end{tabular}

TABLE 6. Effect of oxygen addition on flame speed of methane

\begin{tabular}{c|c||c|c}
\hline $\begin{array}{c}\text { Vol percent } \mathrm{O}_{2} \\
\text { added to air }\end{array}$ & Flame speed fps & $\begin{array}{c}\text { Vol percent } \mathrm{O}_{2} \\
\text { added to air }\end{array}$ & Flame speed fps \\
\hline
\end{tabular}

A. Gas velocity $=4 \mathrm{fps}$

\begin{tabular}{|c|c|c|c|}
\hline \multicolumn{2}{|c|}{ 1. $\frac{\mathrm{Wt} \text { methane }}{\mathrm{Wt} \text { air }+\mathrm{O}_{2}}=0.054$} & \multicolumn{2}{|c|}{ 2. $\frac{\mathrm{Wt} \text { methane }}{\mathrm{Wt} \text { air }+\mathrm{O}_{2}}=0.056$} \\
\hline $\begin{array}{l}0.0 \\
0.5 \\
1.0 \\
2.0 \\
3.0 \\
4.0 \\
5.0 \\
6.0\end{array}$ & $\begin{array}{l}\text { 1. } 074 \\
\text { 1. } 140 \\
\text { 1. } 233 \\
\text { 1. } 267 \\
\text { 1. } 295 \\
\text { 1. } 466 \\
\text { 1. } 472 \\
1.467\end{array}$ & $\begin{array}{l}0.0 \\
0.5 \\
1.0 \\
2.0 \\
3.0 \\
4.0 \\
5.0 \\
6.0\end{array}$ & $\begin{array}{l}\text { 1. } 079 \\
\text { 1. } 223 \\
\text { 1. } 338 \\
\text { 1. } 369 \\
1.482 \\
\text { 1. } 567 \\
1.576\end{array}$ \\
\hline \multicolumn{2}{|c|}{ 3. $\frac{\mathrm{Wt} \text { methane }}{\mathrm{Wt} \text { air }+\mathrm{O}_{2}}=0.058$} & \multicolumn{2}{|c|}{ 4. $\frac{\mathrm{Wt} \text { methane }}{\mathrm{Wt} \text { air }+\mathrm{O}_{2}}=0.060$} \\
\hline $\begin{array}{l}0.0 \\
0.5 \\
1.0 \\
2.0 \\
3.0 \\
4.0 \\
5.0 \\
6.0\end{array}$ & $\begin{array}{l}\text { 1. } 178 \\
\text { 1. } 279 \\
\text { 1. } 340 \\
1.419 \\
\text { 1. } 451 \\
\text { 1. } 564 \\
1.666 \\
1.671\end{array}$ & $\begin{array}{l}0.0 \\
0.5 \\
1.0 \\
2.0 \\
3.0 \\
4.0 \\
5.0 \\
6.0\end{array}$ & $\begin{array}{l}1.214 \\
1.305 \\
1.401 \\
1.459 \\
1.506 \\
1.646 \\
1.755 \\
1.758\end{array}$ \\
\hline \multicolumn{2}{|c|}{ 5. $\frac{\mathrm{Wt} \text { methane }}{\mathrm{Wt} \text { air }+\mathrm{O}_{2}}=0.062$} & \multicolumn{2}{|c|}{ 6. $\frac{\mathrm{Wt} \text { methane }}{\mathrm{Wt} \text { air }+\mathrm{O}_{2}}=0.064$} \\
\hline $\begin{array}{l}0.0 \\
0.5 \\
1.0 \\
2.0 \\
3.0 \\
4.0 \\
5.0 \\
6.0\end{array}$ & $\begin{array}{l}\text { 1. } 232 \\
\text { 1. } 318 \\
\text { 1. } 416 \\
\text { 1. } 501 \\
\text { 1. } 560 \\
\text { 1. } 707 \\
1.825 \\
1.866\end{array}$ & $\begin{array}{l}0.0 \\
0.5 \\
1.0 \\
2.0 \\
3.0 \\
4.0 \\
5.0 \\
6.0\end{array}$ & $\begin{array}{l}1.223 \\
1.322 \\
1.421 \\
1.543 \\
1.590 \\
1.771 \\
1.908 \\
1.909\end{array}$ \\
\hline
\end{tabular}

TABLE 6. Effect of oxygen addition on flame speed of methaneContinued

\begin{tabular}{|c|c|c|c|}
\hline $\begin{array}{l}\text { Vol percent } \mathrm{O}_{3} \\
\text { added to air }\end{array}$ & Flame speed fps & $\begin{array}{l}\text { Vol percent } \mathrm{O}_{3} \\
\text { added to air }\end{array}$ & Flame speed fps \\
\hline \multicolumn{2}{|c|}{ 7. $\frac{\mathrm{Wt} \text { methane }}{\mathrm{Wt} \text { air }+\mathrm{O}_{2}}=0.066$} & \multicolumn{2}{|c|}{ 8. $\frac{\mathrm{Wt} \text { methane }}{\mathrm{Wt} \text { air }+\mathrm{O}_{2}}=0.068$} \\
\hline $\begin{array}{l}0.0 \\
0.5 \\
1.0 \\
2.0 \\
3.0 \\
4.0 \\
5.0\end{array}$ & $\begin{array}{l}1.190 \\
1.411 \\
1.537 \\
1.632 \\
1.872 \\
1.971\end{array}$ & $\begin{array}{l}0.0 \\
0.5 \\
1.0 \\
2.0 \\
3.0 \\
4.0 \\
5.0\end{array}$ & $\begin{array}{l}1.148 \\
1.260 \\
1.392 \\
1.472 \\
1.632 \\
1.815 \\
2.007\end{array}$ \\
\hline \multicolumn{2}{|c|}{ 9. $\frac{\mathrm{Wt} \text { methane }}{\mathrm{Wt} \text { air }+\mathrm{O}_{2}}=0.070$} & \multicolumn{2}{|c|}{ 10. $\frac{\mathrm{Wt} \text { methane }}{\mathrm{Wt} \text { air }+\mathrm{O}_{2}}=0.072$} \\
\hline $\begin{array}{l}0.0 \\
0.5 \\
1.0 \\
2.0 \\
3.0 \\
4.0 \\
5.0\end{array}$ & $\begin{array}{l}1.094 \\
1.200 \\
1.336 \\
1.496 \\
1.629 \\
1.877 \\
2.041\end{array}$ & $\begin{array}{l}0.0 \\
0.5 \\
1.0 \\
2.0 \\
3.0 \\
4.0 \\
5.0\end{array}$ & $\begin{array}{l}1.017 \\
1.117 \\
1.269 \\
1.459 \\
1.615 \\
1.871 \\
2.048\end{array}$ \\
\hline
\end{tabular}

B. Gas velocity $=5 \mathrm{fps}$

\begin{tabular}{|c|c|c|c|}
\hline \multicolumn{2}{|c|}{ 1. $\frac{\mathrm{Wt} \text { methane }}{\mathrm{Wt} \text { air }+\mathrm{O}_{2}}=0.054$} & \multicolumn{2}{|c|}{ 2. $\frac{\mathrm{Wt} \text { methane }}{\mathrm{Wt} \text { air }+\mathrm{O}_{2}}=0.056$} \\
\hline $\begin{array}{l}0.0 \\
0.5 \\
1.0 \\
2.0 \\
3.0 \\
4.0 \\
5.0 \\
6.0\end{array}$ & $\begin{array}{l}\text { 1. } 078 \\
\text { 1. } 147 \\
\text { 1. } 200 \\
\text { 1. } 255 \\
\text { 1. } 347 \\
\text { 1 } 463 \\
\text { 1. } 451 \\
\text { 1. } 480\end{array}$ & $\begin{array}{l}0.0 \\
0.5 \\
1.0 \\
2.0 \\
3.0 \\
4.0 \\
5.0 \\
6.0\end{array}$ & $\begin{array}{l}1.142 \\
1.221 \\
1.258 \\
1.348 \\
1.409 \\
1.510 \\
1.556 \\
1.583\end{array}$ \\
\hline
\end{tabular}

\begin{tabular}{|c|c|c|c|}
\hline \multicolumn{2}{|c|}{ 3. $\frac{\mathrm{Wt} \text { methane }}{\mathrm{Wt} \text { air }+\mathrm{O}_{2}}=0.058$} & \multicolumn{2}{|c|}{ 4. $\frac{\mathrm{Wt} \text { methane }}{\mathrm{Wt} \text { air }+\mathrm{O}_{2}}=0.060$} \\
\hline $\begin{array}{l}0.0 \\
0.5 \\
1.0 \\
2.0 \\
3.0 \\
4.0 \\
5.0 \\
6.0\end{array}$ & $\begin{array}{l}1.178 \\
1.280 \\
1.334 \\
1.425 \\
1.503 \\
1.608 \\
1.645 \\
1.688\end{array}$ & $\begin{array}{l}0.0 \\
0.5 \\
1.0 \\
2.0 \\
3.0 \\
4.0 \\
5.0 \\
6.0\end{array}$ & $\begin{array}{l}1.212 \\
1.312 \\
1.375 \\
1.476 \\
1.564 \\
1.685 \\
1.724 \\
1.764\end{array}$ \\
\hline \multicolumn{2}{|c|}{ 5. $\frac{\mathrm{Wt} \text { methane }}{\mathrm{Wt} \text { air }+\mathrm{O}_{2}}=0.062$} & \multicolumn{2}{|c|}{ 6. $\frac{\mathrm{Wt} \text { methane }}{\mathrm{Wt} \text { air }+\mathrm{O}_{2}}=0.064$} \\
\hline $\begin{array}{l}0.0 \\
0.5 \\
1.0 \\
2.0 \\
3.0 \\
4.0 \\
5.0 \\
6.0\end{array}$ & $\begin{array}{l}1.230 \\
1.327 \\
1.398 \\
1.525 \\
1.621 \\
1.762 \\
1.813 \\
1.849\end{array}$ & $\begin{array}{l}0.0 \\
0.5 \\
1.0 \\
2.0 \\
3.0 \\
4.0 \\
5.0 \\
6.0\end{array}$ & $\begin{array}{l}1.222 \\
1.333 \\
1.392 \\
1.557 \\
1.665 \\
1.771 \\
1.908 \\
1.927\end{array}$ \\
\hline \multicolumn{2}{|c|}{ 7. $\frac{\mathrm{Wt} \text { methane }}{\mathrm{Wt} \text { air }+\mathrm{O}_{2}}=0.066$} & \multicolumn{2}{|c|}{ 8. $\frac{\mathrm{Wt} \text { methane }}{\mathrm{Wt} \text { air }+\mathrm{O}_{2}}=0.068$} \\
\hline $\begin{array}{l}0.0 \\
0.5 \\
1.0 \\
2.0 \\
3.0 \\
4.0 \\
5.0 \\
6.0\end{array}$ & $\begin{array}{l}1.190 \\
1.304 \\
1.389 \\
1.577 \\
1.665 \\
1.851 \\
1.932 \\
1.990\end{array}$ & $\begin{array}{l}0.0 \\
0.5 \\
1.0 \\
2.0 \\
3.0 \\
4.0 \\
5.0 \\
6.0\end{array}$ & $\begin{array}{l}1.148 \\
1.278 \\
1.372 \\
1.558 \\
1.725 \\
1.883 \\
1.990 \\
2.048\end{array}$ \\
\hline
\end{tabular}


TABLE 6. Effect of oxygen addition on flame speed of methaneContinued

\begin{tabular}{|c|c|c|c|}
\hline $\begin{array}{l}\text { Vol percent } \mathrm{O}_{3} \\
\text { added to air }\end{array}$ & Flame speed fps & $\begin{array}{l}\text { Vol percent } \mathrm{O}_{3} \\
\text { added to air }\end{array}$ & Flame speed fps \\
\hline \multicolumn{2}{|c|}{ 9. $\frac{\text { Wt methane }}{\mathrm{Wt} \text { air }+\mathrm{O}_{2}}=0.070$} & \multicolumn{2}{|c|}{ 10. $\frac{\text { Wt metha. } 1 \mathrm{e}}{\mathrm{Wt} \text { air }+\mathrm{O}_{2}}=0.072$} \\
\hline $\begin{array}{l}0.0 \\
0.5 \\
1.0 \\
2.0 \\
3.0 \\
4.0 \\
5.0 \\
6.0\end{array}$ & $\begin{array}{l}1.094 \\
1.206 \\
1.311 \\
1.545 \\
1.710 \\
1.904 \\
2.019 \\
2.099\end{array}$ & $\begin{array}{l}0.0 \\
0.5 \\
1.0 \\
2.0 \\
3.0 \\
4.0 \\
5.0 \\
6.0\end{array}$ & $\begin{array}{l}1.017 \\
1.116 \\
1.234 \\
1.519 \\
1.699 \\
1.897 \\
2.058 \\
2.122\end{array}$ \\
\hline
\end{tabular}

C. Gas velocity $=6 \mathrm{fps}$

\begin{tabular}{|c|c|c|c|}
\hline \multicolumn{2}{|c|}{ 1. $\frac{\mathrm{Wt} \text { methane }}{\mathrm{Wt} \text { air }+\mathrm{O}_{2}}=0.054$} & \multicolumn{2}{|c|}{ 2. $\frac{\text { Wt methane }}{\mathrm{Wt} \text { air }+\mathrm{O}_{2}}=0.056$} \\
\hline $\begin{array}{l}0.0 \\
0.5 \\
1.0 \\
2.0 \\
3.0 \\
4.0 \\
5.0 \\
6.0\end{array}$ & $\begin{array}{l}1.094 \\
1.254 \\
1.316 \\
1.421 \\
1.439 \\
1.497\end{array}$ & $\begin{array}{l}0.0 \\
0.5 \\
1.0 \\
2.0 \\
3.0 \\
4.0 \\
5.0 \\
6.0\end{array}$ & $\begin{array}{l}1.156 \\
1.231 \\
1.262 \\
1.353 \\
1.432 \\
1.499 \\
1.538 \\
1.629\end{array}$ \\
\hline \multicolumn{2}{|c|}{ 3. $\frac{\text { Wt methane }}{\mathrm{Wt} \text { air }+\mathrm{O}_{2}}=0.058$} & \multicolumn{2}{|c|}{ 4. $\frac{\mathrm{Wt} \text { methane }}{\mathrm{Wt} \text { air }+\mathrm{O}_{2}}=0.060$} \\
\hline $\begin{array}{l}0.0 \\
0.5 \\
1.0 \\
2.0 \\
3.0 \\
4.0 \\
5.0 \\
6.0\end{array}$ & $\begin{array}{l}1.188 \\
1.286 \\
1.320 \\
1.418 \\
1.500 \\
1.599 \\
1.636 \\
1.722\end{array}$ & $\begin{array}{l}0.0 \\
0.5 \\
1.0 \\
2.0 \\
3.0 \\
4.0 \\
5.0 \\
6.0\end{array}$ & $\begin{array}{l}1.219 \\
1.313 \\
1.369 \\
1.488 \\
1.571 \\
1.679 \\
1.721 \\
1.809\end{array}$ \\
\hline \multicolumn{2}{|c|}{ 5. $\frac{\text { Wt methane }}{\mathrm{Wt} \text { air }+\mathrm{O}_{2}}=0.062$} & \multicolumn{2}{|c|}{ 6. $\frac{\text { Wt methane }}{\mathrm{Wt} \text { air }+\mathrm{O}_{2}}=0.064$} \\
\hline $\begin{array}{l}0.0 \\
0.5 \\
1.0 \\
2.0 \\
3.0 \\
4.0 \\
5.0 \\
6.0\end{array}$ & $\begin{array}{l}1.233 \\
1.329 \\
1.418 \\
1.519 \\
1.614 \\
1.735 \\
1.781 \\
1.889\end{array}$ & $\begin{array}{l}0.0 \\
0.5 \\
1.0 \\
2.0 \\
3.0 \\
4.0 \\
5.0 \\
6.0\end{array}$ & $\begin{array}{l}1.221 \\
1.329 \\
1.402 \\
1.552 \\
1.650 \\
1.785 \\
1.853 \\
1.955\end{array}$ \\
\hline \multicolumn{2}{|c|}{ 7. $\frac{\mathrm{Wt} \text { methane }}{\mathrm{Wt} \text { air }+\mathrm{O}_{2}}=0.066$} & \multicolumn{2}{|c|}{ 8. $\frac{\text { Wt methane }}{\mathrm{Wt} \text { air }+\mathrm{O}_{2}}=0.068$} \\
\hline $\begin{array}{l}0.0 \\
0.5 \\
1.0 \\
2.0 \\
3.0 \\
4.0 \\
5.0 \\
6.0\end{array}$ & $\begin{array}{l}1.190 \\
1.317 \\
1.386 \\
1.560 \\
1.689 \\
1.852 \\
1.895 \\
2.014\end{array}$ & $\begin{array}{l}0.0 \\
0.5 \\
1.0 \\
2.0 \\
3.0 \\
4.0 \\
5.0 \\
6.0\end{array}$ & $\begin{array}{l}1.148 \\
1.261 \\
1.355 \\
1.569 \\
1.716 \\
1.875 \\
1.947 \\
2.076\end{array}$ \\
\hline \multicolumn{2}{|c|}{ 9. $\frac{\mathrm{Wt} \text { methane }}{\mathrm{Wt} \text { air }+\mathrm{O}_{2}}=0.070$} & \multicolumn{2}{|c|}{ 10. $\frac{\text { Wt methare }}{\mathrm{Wt} \text { air }+\mathrm{O}_{2}}=0.072$} \\
\hline $\begin{array}{l}0.0 \\
0.5 \\
1.0 \\
2.0 \\
3.0 \\
4.0 \\
5.0 \\
6.0\end{array}$ & $\begin{array}{l}1.094 \\
1.213 \\
1.315 \\
1.546 \\
1.716 \\
1.909 \\
1.982 \\
2.115\end{array}$ & $\begin{array}{l}0.0 \\
0.5 \\
1.0 \\
2.0 \\
3.0 \\
4.0 \\
5.0 \\
6.0\end{array}$ & $\begin{array}{l}1.017 \\
1.146 \\
1.256 \\
1.506 \\
1.709 \\
1.914 \\
1.983 \\
2.130\end{array}$ \\
\hline
\end{tabular}

TABLE 6. Effect of oxygen addition on flame speed of methaneContinued

\begin{tabular}{|c|c|c|c|}
\hline $\begin{array}{l}\text { Vol percent } \mathrm{O}_{3} \\
\text { added to air }\end{array}$ & Flame speed fps & $\begin{array}{l}\text { Vol percent } \mathrm{O}_{3} \\
\text { added to air }\end{array}$ & Flame speed fps \\
\hline \multicolumn{4}{|c|}{ D. Gas velocity $=7 \mathrm{fps}$} \\
\hline \multicolumn{2}{|c|}{ 1. $\frac{\mathrm{Wt} \text { methane }}{\mathrm{Wt} \text { air }+\mathrm{O}_{2}}=0.054$} & \multicolumn{2}{|c|}{ 2. $\frac{\mathrm{Wt} \text { methane }}{\mathrm{Wt} \text { air }+\mathrm{O}_{2}}=0.056$} \\
\hline $\begin{array}{l}0.0 \\
0.5 \\
1.0 \\
2.0 \\
3.0 \\
4.0 \\
5.0 \\
6.0\end{array}$ & \begin{tabular}{l}
1.094 \\
\hdashline 1.279 \\
1. 329 \\
1. 436 \\
1. 476
\end{tabular} & $\begin{array}{l}0.0 \\
0.5 \\
1.0 \\
2.0 \\
3.0 \\
4.0 \\
5.0 \\
6.0\end{array}$ & $\begin{array}{l}1.156 \\
-\cdot- \\
1.310 \\
1.372 \\
1.444 \\
1.541 \\
1.588\end{array}$ \\
\hline \multicolumn{2}{|c|}{ 3. $\frac{\mathrm{Wt} \text { methane }}{\mathrm{Wt} \text { air }+\mathrm{O}_{2}}=0.058$} & \multicolumn{2}{|c|}{ 4. $\frac{\mathrm{Wt} \text { methane }}{\mathrm{Wt} \text { air }+\mathrm{O}_{2}}=0.060$} \\
\hline $\begin{array}{l}0.0 \\
0.5 \\
1.0 \\
2.0 \\
3.0 \\
4.0 \\
5.0 \\
6.0\end{array}$ & $\begin{array}{l}\text { 1. } 188 \\
\text { 1. } 231 \\
\text { 1. } 319 \\
\text { 1. } 397 \\
\text { 1. } 456 \\
\text { 1. } 522 \\
\text { 1. } 648 \\
\text { 1. } 708\end{array}$ & $\begin{array}{l}0.0 \\
0.5 \\
1.0 \\
2.0 \\
3.0 \\
4.0 \\
5.0 \\
6.0\end{array}$ & $\begin{array}{l}1.219 \\
1.274 \\
1.373 \\
1.461 \\
1.524 \\
1.620 \\
1.729 \\
1.804\end{array}$ \\
\hline \multicolumn{2}{|c|}{ 5. $\frac{\mathrm{Wt} \text { methane }}{\mathrm{Wt} \text { air }+\mathrm{O}_{2}}=0.062$} & \multicolumn{2}{|c|}{ 6. $\frac{\mathrm{Wt} \text { methane }}{\mathrm{Wt} \text { air }+\mathrm{O}_{2}}=0.064$} \\
\hline $\begin{array}{l}0.0 \\
0.5 \\
1.0 \\
2.0 \\
3.0 \\
4.0 \\
5.0 \\
6.0\end{array}$ & $\begin{array}{l}\text { 1. } 233 \\
\text { 1. } 295 \\
\text { 1. } 400 \\
\text { 1. } 488 \\
\text { 1. } 561 \\
\text { 1. } 678 \\
\text { 1. } 816 \\
1.871\end{array}$ & $\begin{array}{l}0.0 \\
0.5 \\
1.0 \\
2.0 \\
3.0 \\
4.0 \\
5.0 \\
6.0\end{array}$ & $\begin{array}{l}1.223 \\
1.306 \\
1.413 \\
1.522 \\
1.590 \\
1.728 \\
1.825 \\
1.951\end{array}$ \\
\hline \multicolumn{2}{|c|}{ 7. $\frac{\mathrm{Wt} \text { methane }}{\mathrm{Wt} \text { air }+\mathrm{O}_{2}}=0.066$} & \multicolumn{2}{|c|}{ 8. $\frac{\mathrm{Wt} \text { methane }}{\mathrm{Wt} \text { air }+\mathrm{O}_{2}}=0.068$} \\
\hline $\begin{array}{l}0.0 \\
0.5 \\
1.0 \\
2.0 \\
3.0 \\
4.0 \\
5.0 \\
6.0\end{array}$ & $\begin{array}{l}\text { 1. } 190 \\
\text { 1. } 285 \\
\text { 1. } 406 \\
\text { 1. } 527 \\
\text { 1. } 632 \\
\text { 1. } 782 \\
\text { 1. } 918 \\
\text { 2. } 016\end{array}$ & $\begin{array}{l}0.0 \\
0.5 \\
1.0 \\
2.0 \\
3.0 \\
4.0 \\
5.0 \\
6.0\end{array}$ & $\begin{array}{l}1.148 \\
1.253 \\
1.389 \\
1.521 \\
1.632 \\
1.805 \\
1.955 \\
2.069\end{array}$ \\
\hline \multicolumn{2}{|c|}{ 9. $\frac{\text { Wt methane }}{\mathrm{Wt} \mathrm{air}+\mathrm{O}_{2}}=0.070$} & \multicolumn{2}{|c|}{ 10. $\frac{\mathrm{Wt} \text { methane }}{\mathrm{Wt} \text { air }+\mathrm{O}_{2}}=0.072$} \\
\hline $\begin{array}{l}0.0 \\
0.5 \\
1.0 \\
2.0 \\
3.0 \\
4.0 \\
5.0 \\
6.0\end{array}$ & $\begin{array}{l}\text { 1. } 094 \\
\text { 1. } 194 \\
\text { 1. } 347 \\
\text { 1. } 490 \\
\text { 1. } 680 \\
\text { 1. } 816 \\
\text { 1. } 983 \\
\text { 2. } 099\end{array}$ & $\begin{array}{l}0.0 \\
0.5 \\
1.0 \\
2.0 \\
3.0 \\
4.0 \\
5.0 \\
6.0\end{array}$ & $\begin{array}{l}1.017 \\
1.157 \\
1.304 \\
1.458 \\
1.666 \\
1.827 \\
1.992 \\
2.124\end{array}$ \\
\hline
\end{tabular}

\section{Conclusions}

Both perchloryl fluoride and oxygen increase the flame speed when added to burning mixtures of methane and air, and the effect of perchloryl fluoride, on a molar basis, is greater than that of oxygen. However, preheating the methane-air mixture moderately, up to temperatures of $330^{\circ} \mathrm{F}$, is more effective in increasing flame speeds than the addition of small amounts of either oxidant. The mixture ratio at which maximum flame speed occurs is dis- 
placed bevond 0.072 by only small additions of either perchloryl fluoride or oxygen. In the range of addition of oxidants covered in this report, the maximum flame speed increases linearly with the addition of either perchloryl fluoride or oxygen, although at different rates.

\section{References}

[1] A. Engelbrecht and H. Atzwanger, Monatsh. Chem. 83, 1087 (1952).

[2] Private communication from the Pennsylvania Salt Manufacturing Co.

[3] C. Neugebauer and J. L. Margrave, J. Am. Chem. Soc. 79, 1138 (1957).

[4] R. M. Lodwig and J. L. Margrave, Combustion and Flame 3, 147 (1959)

[5] A. G. Gaydon and H. G. Wolfhard, Flames, their structure, radiation and temperature, Chapman and Hall, Ltd., London, p. 68-72 (1960).
[6] J. W. Linnett, Fourth Symposium (International) on Combustion, 1952. Williams and Wilkins, Baltimore, Md., p. 20 (1953).

[7] C. Halpern, J. Research NBS 60, 535 (1958) RP2867.

[8] B. Lewis and G. von Elbe, Combustion, flames and explosions of gases. Academic Press, Inc., N.Y., p. 465 (1951).

[9] Reported in Lewis and von Elbe, op. cit., p. 466.

[10] E. F. Fiock, Physical measurements in gas dynamics and combustion, Princeton University Press, Princeton, N.J., p. 433 (1954).

[11] Reported in Lewis and von Elbe, op. cit., p. 467.

[12] J. M. Singer, J. Grumer, and E. B. Cook, Proc. gas dynamics symp. aerothermodynamics, 1955. Northwestern University, Evanston, Ill., p. 139 (1956).

(Paper 65A6-134) 


\title{
Publications of the National Bureau of Standards*
}

\author{
(Including papers in outside journals)
}

\section{Selected Abstracts}

\begin{abstract}
Analysis of pyrolyzates of polystyrene and Poly (methyl methacrylate) by gas chromatography, $F$. A. Lehmann and G. M. Brauer, Anal. Chem. 33, No. 6, 673-676 (May 1961).

Pyrolyzates of polystyrene and poly(methyl methacrylate) were studied at temperatures from 400 to $1100^{\circ} \mathrm{C}$ using gas chromatographic techniques. A small Vycor boat surrounded by a platinum heating coil was placed in the inlet system of the chromatograph. A 2 to $3 \mathrm{mg}$ sample was degraded at a series of temperatures which were measured by means of a thermocouple placed in an indentation at the bottom of the boat. Helium carried the volatile products into the heated column of the chromatograph. Analyses of the products could usually be accomplished with a reproducibility of better than $1 \%$. Polystyrene degraded mainly to monomer below $700^{\circ} \mathrm{C}$ and poly(methyl methacrylate) behaves similarly below $450^{\circ} \mathrm{C}$. At higher temperatures some gaseous and liquid decomposition products are formed which were identified by their characteristic retention times. The technique uses inexpensive equipment and is generally applicable for the study of the thermal stability of polymers and their pyrolyzates. Results obtained are very helpful in identifying reaction products and in the elucidation of degradation mechanisms over wide temperature ranges. The procedure is also useful in the rapid characterization and quantitative estimation of the composition of complex copolymer systems.
\end{abstract}

Microwave spectrum of isobutylene. Dipole moment, internal barrier, equilibrium conformation, structure, $V . W$. Laurie, J. Chem. Phys. 34, No. 5, 1516-1519 (May 1961).

The microwave spectra of isobutylene and isobutylene- $d-3$ have been studied in the region 17-36 kMc. Observed rotational constants (Mc) for isobutylene are $a_{0}=9133.32$, $b_{0}=8381.75, c_{0}=4615.99$; for $s y m$-isobutylene- $d-3, a_{0}=9132.59$, $b_{0}=7788.98, \quad c_{0}=4431.05 ; \quad$ for asym-isobutylene- $d-3$, $a_{0}=8819.46, \quad b_{0}=7981.08, \quad c_{0}=4469.59$. The most likely structure compatible with these data is $r_{c-c}=1.50_{7} \mathrm{~A}, r_{c-c}$ $=1.34 \mathrm{~A}, \quad r_{\mathrm{CH}}($ methyl $)=1.08_{5} \mathrm{~A}, r_{\mathrm{CH}}($ ethylenic $)=1.08_{6} \mathrm{~A}$, $<\mathrm{Me}-\mathrm{C}-\mathrm{Me}=115.9^{\circ},<\mathrm{HCH}$ (methyl) $=108^{\circ},<\mathrm{HCH}$ (ethylenic $)=117.5^{\circ}$. Measurements of the Stark effect show that the dipole moment $\mu=0.503+0.009 D$. Fine structure leads to a barrier height hindering internal rotation of the methyl groups of $2.21 \mathrm{kcal} / \mathrm{mole}$. The equilibrium conformation is $\mathrm{C}_{2} \nu$ with two methyl hydrogens in the plane of the carbon atoms and the $\mathrm{CH}$ bonds pointing away from the symmetry axis. It is concluded that although CC single bonds are shortened when adjacent to a double bond, the double bond length is probably not much affected by the presence of an adjacent $\mathrm{CH}_{3}$ group.

Nonequilibrium processes in isotopically disordered crystals. Dependence on degree of order, R. Zwanzig, J. Math. Phys. 2, No. 3, 370-372 (May-June 1961).

Maradudin, Weiss, and Jepsen have shown that approximate normal modes of isotopically disordered crystals decay irreversibly when the arrangement of isotopes is completely random. We generalize their results to crystals with an arbitrary degree of order. In particular, we show that irreversible behavior occurs whenever the spatial correlation between isotopic species extends over a fixed finite range, as the size of the crystal tends to infinity.

Intermolecular forces from optical spectra of impurities in molecular crystals, $R$. W. Zwanzig, Molecular Phys. 3, No. 4, 305-311 (July 1960).

A procedure is outlined by which one can get information about the effects of electronic excitation on the interactions between molecules. The absorption and emission spectra of impurities in molecular crystals, and specifically the mean positions and widths of the lines are required as functions of temperature. A theory of M. Lax is used to relate mean position and width to intermolecular forces. The preliminary treatment of this problem by McCarty and Robinson is evaluated and extended: their procedure is expected to be valid for very narrow lines only.

Spin-lattice relaxation in cerous magnesium nitrate, $R$. $P$. Hudson and R. S. Kaeser, Il Nuovo Cimento 19, Serie $X$, 1275-1277 (Mar. 16, 1961).

Measurements of the spin-lattice relaxation time, $\tau$, at low audiofrequencies for cerous magnesium nitrate in the region of $2^{\circ} \mathrm{K}$ confirm the existence of an excited level at about $25 \mathrm{~cm}^{-1}$. The consequent exponential dependence of $\tau$ on temperature, $T$, gives way below $2^{\circ} \mathrm{K}$ to a term varying as $T^{-5}$. It seems likely that the latter arises from the combined effect of a direct relaxation process $\left(T^{-1}\right)$ and a "Ramantype" process $\left(T^{-7}\right)$, here making comparable contributions to the relaxation at an unusually low temperature.

The photolysis of ethyl vinyl ether, E. Murad, J. Am. Chem. Soc. 83, 1327-1330 (1961).

This direct photolysis of ethyl vinyl ether has been studied and the primary photochemical processes determined. The same processes have been found to occur in the mercury-sensitized decomposition, viz,

$$
\begin{aligned}
\mathrm{CH}_{2}=\mathrm{CHOCH}_{2} \mathrm{CH}_{3}+\mathrm{h} \nu & \rightarrow \mathrm{C}_{2} \mathrm{H}_{5}+\mathrm{CH}_{2}=\mathrm{CHO} \\
& \rightarrow \mathrm{C}_{2} \mathrm{H}_{4}+\mathrm{CH}_{3} \mathrm{CHO}
\end{aligned}
$$

An additional primary process was found to occur in the $\mathrm{Co}^{60} \gamma$-radiolysis, viz,

$$
\mathrm{CH}_{2}=\mathrm{CHOCH}_{2} \mathrm{CH}_{3}+\gamma \rightarrow \mathrm{C}_{2} \mathrm{H}_{5} \mathrm{O}+\mathrm{CH}_{2}=\mathrm{CH}
$$

The configuration of the excited state leading to process II is suggested to be a six-membered ring intermediate.

Vapor phase gamma-radiolysis of azomethane, L. J. Stief and P. Ausloos, J. Phys. Chem. 65, 877-881 (1961).

The effect of scavengers, pressure, temperature, and added zenon on the vapor phase $\gamma$-radiolysis of azomethane has been investigated. Most of the results can be explained on the basis of free radical reactions similar to those occurring in the photolysis of azomethane. Values for the ratio of rate constants $k^{1} / k_{21}^{1 / 2}$ for the reactions

$$
\begin{gathered}
\text { If } \mathrm{CH}_{3}+\mathrm{CH}_{3} \mathrm{~N}_{2} \mathrm{CH}_{3} \rightarrow \mathrm{CH}_{4}+\mathrm{CH}_{2} \mathrm{~N}_{2} \mathrm{CH}_{3} \\
\mathrm{CH}_{3}+\mathrm{CH}_{3} \rightarrow \mathrm{C}_{2} \mathrm{H}_{3}
\end{gathered}
$$

determined from the radiolysis data are in excellent agreement with values based on photolysis experiments, indicating that methane and ethane are formed by the reactions of thermalized methyl radicals. The results are best explained on the basis of the decomposition of an electronically excited molecule, formed either by direct excitation or by ionization followed by neutralization. Ion decomposition and ionmolecule reactions of the usual type are shown to be inconsistent with the results.

Electron interferometer studies of iron whiskers, $H . A$. Fowler, L. Marton, J. A. Simpson, and J. A. Suddeth, J. Appl. Phys. 32, No. 6, 1153 (June 1961).

Electron interference patterns have been obtained by using an iron whisker as the electrostatic fiber of a Möllenstadt (1) interferometer. The tilted fringes in the shadow pattern resemble those observed by Chambers (2) and show the relationship between flux leakage and geometrical shape of the whisker. Chambers' general model of the flux configuration is confirmed. 
(1) G. Möllenstadt and H. Düker, Z. Physik 145, 377 (1956);

(2) R. G. Chambers, Phys. Rev. Letters 5, 3 (July 1960).

Dissociation constant of the protonated acid form of 2-amino2-(hydroxymethyl) -1, 3-propanediol [tris (hydroxymethyl)aminomethane] and related thermodynamic quantities from 0 to $50^{\circ}, R$. G. Bates and H. B. Hetzer, J. Phys. Chem. 65, $667-671$ (1961).

The thermodynamic dissociation constant $\left(K_{b h}\right)$ of the protonated form $\left(\mathrm{BH}^{+}\right)$of 2-amino-2-(hydroxymethyl)-1,3propanediol [tris (hydroxymethyl)aminomethane] has been determined at 11 temperatures from 0 to $50^{\circ}$ by measurement of the electromotive force of hydrogen-silver chloride cells without liquid junction. The results are given by the equation

$$
-\log K_{b h}=\frac{2981.4}{T}-3.5888+0.005571 T,
$$

where $T$ is in ${ }^{\circ} \mathrm{K}$. The changes of free energy, enthalpy, entropy, and heat capacity were computed for the dissociation of the cation acid $\mathrm{BH}^{+}$in the standard state, as well as for the basic dissociation, $\mathrm{B}+\mathrm{H}_{2} \mathrm{O}=\mathrm{BH}^{+}+\mathrm{OH}^{-}$.

For the acidic dissociation at $25^{\circ}, \Delta G^{\circ}$ is $46,075 \mathrm{j} \mathrm{mole}^{-1}$, $\Delta H^{\circ}$ is $47,600 \mathrm{j} \mathrm{mole}^{-1}, \Delta S^{\circ}=5.1 \mathrm{j} \mathrm{deg}^{-1} \mathrm{~mole}^{-1}$, and $\Delta C_{p}^{\circ}$ is $-64 \mathrm{j} \mathrm{deg}^{-1}$ mole $^{-1}$. The activity-coefficient term is consistent with an ion-size parameter of zero; hence, incomplete dissociation of the salt $\mathrm{BHCl}$ is suggested. The entropy changes for the isoelectric dissociation of cation acids $\left(\mathrm{BH}^{+}\right)$appear to indicate restrictive orientation of solvent molecules about the ions and the attendant hindrance of free rotation about carboncarbon bonds. This simple picture is, however, unable to account satisfactorily for the observed changes in heat capacity.

Hyperfine structure and isotope shifts in the 2537-A line of mercury, W. G. Schweitzer, Jr., J. Opt. Soc. Am. 51, No. 6, 692-693 (June 1961).

The hyperfine structure and isotope shifts in the $2537 \AA$ line of natural mercury have been measured with high precision using interferometric techniques on the absorption spectrum of an atomic beam.

Paramagnetic substances for nuclear orientation, $R . P$. Hudson, Prog. in Cryogenics, 3, 99-127 (1961).

An account is given of the magnetic and thermal properties of the substances which have proved most useful for producing nuclear orientation at very low temperatures. This is prefaced by a discussion of factors affecting the choice of substance.

Age to indium resonance for D-D neutrons in heavy water, V. Spiegel, Jr., and A. C. B. Richardson, Nuclear Sci. and Eng. 10, No. 1, 11-15 (1961).

The neutron age to the $1.44 \mathrm{ev}$ resonance in indium has been determined from activation measurements for a $D(d, n) \mathrm{He}^{3}$ neutron source in heavy water. Appropriately averaged and corrected indium foil activities yield the value $115.5+1.4$ $\mathrm{cm}^{2}$ for the age in an infinite medium. Independent theoretical calculations for exactly this experimental arrangement by Cooper and for a comparable case by Sullivan both yield values in agreement with this experimental result. It appears, therefore, that there is at present no discrepancy between theory and experiment for the age of $2-3 \mathrm{Mev}$ neutrons in heavy water.

Magnetic properties and optical absorption spectrum of $\mathbf{K}_{2} \mathbf{R e C l}_{6}, J$. C. Eisenstein, J. Chem. Phys. 34, No. 5, 16281648 (May 1961).

A phenomenological theory of certain physical properties of the $\left(\mathrm{ReCl}_{6}\right)^{2-}$ complex ion is presented here. The ion can be described in terms of a set of parameters which includes the strength of the octahedral ligand field, the Coulomb integrals, the spin-orbit coupling constant and the orbital reduction factors. The interaction matrices for the three electron system are given in algebraic form. With an appropriate choice of the parameters the eigenvalues of these matrices are in fair agreement with the observed energy levels of the system. The effect of a departure from strict octahedral symmetry is discussed. The matrix elements of the magnetic moment operator are also given in algebraic form and then used in a calculation of the susceptibility. The discripancy between theory and experiment is attributed to a superexchange interaction with leads, at sufficiently low temperatures, to antiferromagnetism of the compound. Finally, the results of paramagnetic resonance experiments on $\mathrm{K}_{2} \mathrm{ReCl}_{6}$ are considered.

Calculations of the neutron age in water and heavy water for D-D sources, J. W. Cooper, Nuclear Sci. and Eng. 10, No. 1, 1-10 (May 1961)

Calculations of the age to indium resonance of neutrons from the reaction $D(d, n) \mathrm{He}^{3}$ in water and heavy water have been performed by the Monte Carlo method. The calculations were designed to simulate the experiments of Spiegel et al. The effect of the duct used to lead the deuteron beam into the medium was investigated. The computations show agreement with the experimental results and with a similar calculation performed by C. R. Mullin.

Magnetic susceptibility of cerous magnesium nitrate, $R$. $P$. Hudson and $W$. R. Hosler, Phys. Rev. 122, No. 5, 1417-1420 (June 1961).

The most striking features of the behavior of cerous magnesium nitrate at liquid helium temperatures-extremely anisotropic susceptibility with $g_{\|} \approx 0$, a large temperatureindependent term in $\chi_{\perp}$, the spin-lattice relaxation time varying as the twelfth or higher power of $T$ in the region of $2^{\circ} \mathrm{K}$ - have until very recently received no detailed explanation. A measurement of $\chi_{\perp}$ between $4^{\circ} \mathbf{K}$ and $300^{\circ} \mathrm{K}$ was undertaken to elicit information on the energies of the excited doublets within the $J=5 / 2$ ground multiplet, and thus to provide a guide for the reassessment of the crystal field parameters. We find $\delta_{1}$ and $\delta_{2}$ to be 30 and (roughly) $200 \mathrm{~cm}^{-1}$ respectively, in contrast to the 113 and $150 \mathrm{~cm}^{-1}$ of of existing theory. The results are at variance with the published data for $\chi_{\perp}$ above $85^{\circ} \mathrm{K}$, but are in fairly good agreement with the recent findings of Leask and Wolf at low temperatures.

Use of gaussian orbitals for atoms-in-molecule calculations, M. Kraus, J. Chem. Phys. 34, No.2,692-693 (February 1961). The use of Gaussian functions is proposed for "atoms-inmolecule" calculations and applied for the case of $\mathrm{H}_{2}$. Reasonably accurate results are obtained for the equilibrium internuclear distance and the binding energy. The results justify further work with more complicated systems.

Measurement of minority carrier lifetime in $\mathrm{SiC}$ by a novel electroluminescent method, G. G. Harman and R. L. Raybold, J. Appl. Phys. 32, No. 6, 1168-1169 (June 1961).

A new method of measuring the lifetime of minority carriers in large energy gap semiconductors is described. This consists of injecting and subsequently extracting carriers with a sine wave generator while monitoring the average carrier concentration by observing the emitted recombination radiation. When $1 /$ f seconds is comparable to the lifetime this average emitted light falls off sharply. The method was applied to silicon carbide crystals and it was found that the majority had lifetimes $\sim 10^{-8}$ sec but a few were as long as $10^{-6}$ sec.

Absolute measurement of $\mathrm{W}$ for $\mathrm{Po}^{210}$ alpha particles in air, nitrogen and carbon dioxide, $Z$. Bay, $P$. A. Newman, and $H$. H. Seliger, Radiation Research 14, No. 5, 551-565 (May 1961). The average energy $(W)$ expended in producing an ion pair in air nitrogen, and carbon dioxide was measured for the alpha particles of $\mathrm{PO}^{210}$ while they are slowed down from their initial energy (5.305 Mev) to zero energy. The ionization chamber was used simultaneously in these measurements as an integrating current-measuring chamber and as a pulse ionization chamber. In this way, the ratio of the total charge measured to the total number of pulses counted is independent of the random statistical fluctuations in the emission rate of the low activity sources used. A small part of the chamber wall (which served as the high-voltage electrode) holding the alpha source and separated AC-wise from the rest of the chamber was used for pulse counting. A careful investigation of 
the various factors involved helped to obtain a final accuracy of the measurements of about $0.1 \%$. The results are $W_{\alpha_{\mathrm{air}}}=$ $35.2 \pm 0.1 \mathrm{ev} /$ ion pair, $W_{\alpha N_{2}}=36.4 \pm 0.1 \mathrm{ev} / \mathrm{ion}$ pair and $W_{\alpha} \mathrm{CO}_{2}=34.3 \pm 0.2 \mathrm{ev} /$ ion pair. The indicated uncertainties include the standard errors of the respective means plus an allowance for other known sources of possible errors.

The structure of the monoclinic form of sodium tetrametaphosphate tetrahydrate, H. M. Ondik, C. H. MacGillavry, and S. Block, Acta. Cryst. 14, 555 (1961).

Crystals of monoclinic sodium tetrametaphosphate tetrahydrate, $\mathrm{Na}_{4} \mathrm{P}_{4} \mathrm{O}_{12} \cdot 4 \mathrm{H}_{2} \mathrm{O}$, are needlelike in habit, elongated along $c$. The unit cell dimensions are $a=9.667 \pm 0.002$, $b=12.358 \pm 0.002, c=6.170 \pm 0.001 \AA, \beta=92^{\circ} 16^{\prime} \pm 10^{\prime}$; space group $\mathrm{P} 2_{1} / a, Z=2$. Two sets of three-dimensional $\mathrm{X}$-ray diffraction data were collected with $\mathrm{CuK}_{\alpha}$ radiation using Weissenberg multiple-film techniques and the intensities were measured photometrically. The structure was determined by a combination of three-dimensional Patterson and electron-density syntheses, and a complete-matrix leastsquares reduction of data. The final $R$ value for 831 nonzero reflections is 0.108 . Individual isotropic temperature factors were used.

The tetrametaphosphate rings are on centers of symmetry The sodium ions are coordinated by six oxygen atoms in roughly octahedral configuration. One sodium ion links the rings in a network parallel to the $a b$ plane. The other alternates with the rings linking them along $c$.

Mass spectrometric study of cyanogen and cyanoacetylenes, V. H. Dibeler, R. M. Reese, and J. L. Franklin, J. Am. Chem. Soc. 83, 1813-1818 (1961).

Relative abundances and appearance potentials are reported for the principal ions in the mass spectra of cyanogen, cyanoacetylene, dicyanoacetylene, and dicyanodiacetylene. Heats of formation of radicals and radical ions are calculated and the energetically probable ionization-dissociation processes are tabulated. Carbon-carbon and carbon-nitrogen bond dissociation energies are calculated for various ionized and neutral molecular species.

Energy levels and magnetic dipole transitions in the $4 p^{4}$ ground con figuration of singly ionized atomic bromine $(\mathrm{Br}$ II), W. C. Martin and J. L. Tech, J. Opt. Soc. Am. 51, No. 6, 591-594 (June 1961).

Improved values of the previously known $\mathrm{Br}$ II energy levels relative to the $4 p^{4}{ }^{3} p_{2}$ ground level at $0.0 \mathrm{~cm}^{-1}$, have been obtained from preliminary observations in the vacuum ultraviolet region. The position of the $4 p^{4}{ }^{1} S_{0}$ level was found from the "forbidden" transition, $4 p^{4}{ }^{3} p_{1}-4 p_{4}{ }^{1} S_{0}$ at 4042.42 A. An observation of the transverse Zeeman effect for this line yields a Lorentz triplet with the usual polarizations reversed and approximately the expected splitting. It is thus confirmed that the line arises from magnetic-dipole radiation. A similar line at $8269.65 \mathrm{~A}$, due to the transition $4 p^{4}{ }^{3} p_{2}-$ $4 p^{4}{ }^{1} D_{2}$, shows hyperfine-structure broadening consistent with the calculated structure. New values for the excited levels of $4 p^{4}$ are: ${ }^{3} p_{1}=3136.4 \mathrm{~cm}^{-1},{ }^{3} p_{0}=3837.5 \mathrm{~cm}^{-1},{ }^{1} D_{2}=12089.1$ $\mathrm{cm}^{-1}$, and ${ }^{1} S_{0}=27867.1 \mathrm{~cm}^{-1}$. The values for the levels of all configurations above $4 p^{4}$, as given in Atomic Energy Levels, Vol. II, should be reduced by $5.9 \pm 0.5 \mathrm{~cm}^{-1}$. Previous values for the levels of $4 p^{4}$ were also in error by several $\mathrm{cm}^{-1}$, and earlier evidence for the position of $4 p^{4}{ }^{1} S_{0}$ was inconclusive. Intermediate-coupling parameter values which yield a good fit for $\mathrm{Br}$ II $4 p^{4}$ are $F_{2}(4 p, 4 p)=1690 \mathrm{~cm}^{-1}$ and $\zeta(4 p)=$ $2800 \mathrm{~cm}^{-1}$.

An improved structure determination for vinyl fluoride, D. R. Lide, Jr., and D. Christensen, Spectrochim. Acta 17, 665-668 (1961).

The previously published rotational constants of 8 isotopic species of vinyl fluoride have been used to calculate an $r_{s}$ (substitution) structure for the molecule. The results are: $r(\mathrm{CC})=1.329 \pm 0.006 \mathrm{~A}, r(\mathrm{CF})=1.347 \pm 0.009 \mathrm{~A}, \Varangle \mathrm{FCC}=$ $120.8^{\circ} \pm 0.3^{\circ}, \Varangle \mathrm{FCH}=110^{\circ} \pm 1^{\circ}, r(\mathrm{CH})=1.032 \pm 0.004 \mathrm{~A}$; in the $=\mathrm{CH}_{2}$ group: $r(\mathrm{CH})=1.077 \pm 0.003 \mathrm{~A}$ and $\Varangle \mathrm{HCC}=$ $119.0^{\circ} \pm 0.3^{\circ}$ for the $\mathrm{CH}$ bond trans to $F ; r(\mathrm{CH})=1.087 \pm$ $0.002 \mathrm{~A}$ and $\Varangle \mathrm{HCC}=120.9^{\circ} \pm 0.3^{\circ}$ for the $\mathrm{CH}$ bond cis to $F$

\section{Other NBS Publications}

Journal of Research 65C (Eng. and Instr.) No. 4, (Oct.-Dec. 1961) 75 cents.

A new airglow photometer. C. M. Purdy, L. R. Megill, and F. E. Roach.

A guide to the use of the modified reflectometer technique of VSWR measurement. W. J. Anson.

An X-ray diffractometer cryostat providing temperature control in the range 4 to $300^{\circ} \mathrm{K}$. F. A. Mauer and L. H. Bolz.

Apparatus for determination of pressure-density-temperature relations and specific heats of hydrogen to 350 atmospheres at temperatures above $14{ }^{\circ} \mathrm{K}$. Robert D. Goodwin.

The use of a thermistor for detecting eluent fronts in liquidsolid chromatography. G. S. Ross.

Radiation field from a circular disk source. J. H. Hubbell, R. L. Bach, and R. J. Herbold.

The Bauschinger effect and residual microstresses in alpha brass. C. J. Newton.

A study by polarization techniques of the corrosion rates of aluminum and steel underground for sixteen months. W. J. Schwerdtfeger.

Journal of Research 65D, (Radio Prop.) No. 6, (Nov.-Dec. 1961) 70 cents.

The solar wind. E. N. Parker.

Attentuation coefficients for propagation at very low frequencies (VLF) during a sudden ionospheric disturbance (SID). E. T. Pierce.

Dipole radiation in a conducting half space. R. K. Moore and W. E. Blair.

Reliability of atmospheric radio noise predictions. J. R. Herman.

Effects of the ionosphere on VLF navigational aids. W. T. Blackband.

On the spectrum of terrestrial radio noise at extremely low frequencies. H. R. Raemer.

The nonsingular embedding of transition processes within a more general framework of coupled variables. J. Heading.

Worldwide VLF standard frequency and time signal broadcasting. A. D. Watt, R. W. Plush, W. W. Brown, and A. H. Morgan.

Design of panoramic ionospheric resources. L. H. Heisler and L. D. Wilson.

A quick method for estimating the stage of the sunspot cycle. W. B. Chadwick.

Measurements of low-angle radiation from a monopole. A. C. Wilson.

Capacities of stacks in sanitary drainage systems for buildings, R. S. Wyly and H. N. Eaton, NBS Mono. 31 (1961), 35 cents.

Mean electron density variations of the quiet ionosphere 4 June 1959, J. W. Wright, L. R. Wescott, and D. J. Brown, NBS TN40-4 (PB151399-4) (1961) $\$ 1.50$

Bibliography on ionospheric propagation of radio waves (1923-1960), W. Nupen, NBS TN84 (PB161585) (1961) $\$ 7.00$.

Collisions of liquid drops with liquids, O. G. Engel, NBS TN89 (PB161590) (1961) \$1.00.

Determination of the $\mathrm{K}$ fluorescence yield of argon by proportional-counter spectrometry, C. Godeau, NBS TN91 (PB161592) (1961) 50 cents.

On the climatology of ground-based radio ducts and associated fading regions, E. J. Dutton, NBS TN96 (PB161597) (1961) $\$ 1.75$.

A fixed frequency, 9.1 Gc, field intensity recording receiver with extremely narrow bandwidth, R. W. Hubbard and J. V. Cateora, NBS TN107 (PB161608) (1961) 75 cents.

Data reduction instrumentation for radio propagation research, W. E. Johnson, NBS TN111 (PB161612) (1961) $\$ 1.00$.

The glass electrode, R. G. Bates, Book, Reference Electrodes, edited by D. J. G. Ives and G. J. Janz (Academic Press Inc., New York, N.Y.), ch. 5, 231-269 (1961). 
A recalibration of the NBS standard thermal neutron flux, E. R. Mosburg, Jr., and W. M. Murphey, J. Nuclear Eng. (Reactor Science and Technology Pt.) 14, 25-30 (1961).

The relationship of low-height ionosonde echoes to auroralzone absorption and VHF $D$ scatter, J. K. Olesen and J. W. Wright, J. Geophys. Research 66, No. 4, 1127-1134 (Apr. 1961).

Trends in the technology of automatic data processing, S. N. Alexander, AMA Report 43, 38-42 (1960).

Use of the bulge test for determining mechanical properties of stainless steel foil, D. B. Ballard, Materials Research and Standards 1, No. 6, 471-473 (June 1961)

Some comments on the use and preparation of hard gallium alloys, G. G. Harman, Rev. Sci. Instr. 32, No. 4, 465-466 (Apr. 1961).

On the nature of equatorial spread $F, \mathrm{R}$. Cohen and K. L. Bowles, J. Geophys. Research 66, No. 4, 1081-1106 (Apr. 1961).

A study of the chemical reactions in kilning bone char, F. G. Carpenter, and V. R. Deitz, Proc. 6th Tech. Session on Bone Char 1959, p. 321 (Bone Char Research Project Inc., Charlestown, Massachusetts, 1961).

Rate of vaporization of refractory substances, R. F. Walker, J. Efimnokof, and N. L. Lofgren, Book, Physical Chemistry in Aerodynamics and Space Flight (Pergamon Press, Inc., London, England), p. 24-30 (1961).

A note on phase velocity of VLF radio waves, J. R. Wait and K. P. Spies, J. Geophys. Research 66, No. 3, 992-993 (Mar. 1961).

On the excitation of electromagnetic surface waves on a curved surface, J. R. Wait, IRE Trans. Ant. Prop. AP-8, No. 4, 445-448 (July 1960).

Solubility product phenomena in hydroxyapatite-water systems, R. M. Rootare, V. R. Deitz, and F. G. Carpenter, Proc. 6th Tech. Session on Bone Char 1959, p. 287 (Bone Char Research Project Inc., Charlestown, Massachusetts 1961).

Reproducibilities of carbon and germanium thermometers at $4.2^{\circ}$ K, M. H. Edlow and H. H. Plumb, Proc, Cryogenic Eng. Conf., Boulder, Colo., Aug. 1960, 6, 542-547 (1961).

The automatic chloride titrator in the analysis of some ionic constituents, M. D. Peiperl, F. G. Carpenter, and V. R. Deitz, Proc. 6th Tech. Session on Bone Char 1959, p. 53 (Bone Char Research Project Inc., Charlestown Massachusetts 1961).

Changes in dentures during storage in water and in service, J. B. Woefel, G. C. Paffenbarger, and W. T. Sweeney, J. Am. Dental Assoc, 62, No. 6, 643-657 (June 1961).

Solar disturbances and radio communication forecasts, J. F. Brockman, Sky and Telescope XXI, No. 6. 322-326 (June 1961).

Long-distance one-hop $F_{1}$ propagation through the auroral zone, L. H. Tveten, J. Geophys. Research 66, No. 6, 16831684 (June 1961).

Resonators for millimeter and submillimeter wavelengths, W. Culshaw, IRE Trans. Microwave Theory and Tech. MTT-9, No. 2, 135-144 (March 1961).

A five-attribute system of describing visual appearance, D. B. Judd, Am. Soc. Testing Materials Spec. Tech. Pub. No. 297 (1961)

Circumferentially uniform electroplating of tube bores, $\mathrm{J} P$. Young and H. I. Salmon, Plating 48, No. 7, 763; 787-8 (July 1961).

Solar flare effects in the $F$ region of the ionosphere, R. W. Knacht and K. Davies, Nature 190, No. 4778, 797-798 (May 1961).

Convective circulation in water induced by evaporation cooling, W. G. Spangenberg and W. R. Rowland, Phys. of Fluids 4, No. 6, 743-750 (June 1961).

Photoionization heating in the $F$ region of the atmosphere, D. C. Hunt and T. E. Van Zandt, J. Geophys. Research 66, No. 6, 1673-1682 (June 1961).
Identification of fiber blends by infrared spectroscopy' M. K. Wharton and F. H. Forziati, Am. Dyestuff Rep. 50, No. 14, 33-36 (July 1961).

Residual losses in a guard-ring micrometer-electrode holder for solid-disk dialectric specimens, A. H. Scott and W. P. Harris, 1960 Annual Report, Conference on Electrical Insulation, Natl. Acad. Sci.-Natl. Research Council, 11-17 (1961)

Comparative $p \mathrm{H}$ measurements on papers by water extraction and glass electrode spot tests, J. H. Flynn and L. E. Smith, Tappi 44, No. 3, 223-228 (March 1961).

A comparison between theoretical and experimental data on phase velocity of VLF radio waves, J. R. Wait, Proc. IRE 49, No. 6, 1089-1090 (June 1951).

A diffraction theory for LF sky-wave propagation, J. R. Wait, J. Geophys. Research 66, No. 6, 1713-1724 (June 1961); An additional note, J. R. Wait and A. M. Conda, J. Geophys. Research 66, No. 6, 1725-1729 (June 1961).

An analysis of errors in the calibration of electric instruments, F. L. Hermach, Communication and Electronics, 1-6 (May 1961).

Incoherent scattering by free electrons as a technique for studying the ionosphere and exosphere; Some observations and theoretical considerations, K. L. Bowles, pp. 223-243 of AGARDograph 42, The upper atmosphere above $F_{2}$ maximum. North Atlantic Treaty Organization, Advisory Group for Aeronautical Research and Development, Avionics Panel. Papers presented at the Symposium of the Ionospheric Research Committee, Paris, France, May 1959.

A $p \mathrm{H}$ standard for blood and other physiologic media, V. E. Bower, M. Paabo, and R. G. Bates, Clin. Chem. 7, No. 3, 292 (June 1961).

Evidence on the laminar nature of the exosphere obtained by means of guided high-frequency wave propagation, R. M. Galley and W. F. Utlaut, Phys. Rev. Letters 6, No. 11, 591-594 (June 1, 1961).

Comparison of the ionization produced in air by alpha particles near $5 \mathrm{Mev}$ and by beta particles, Z. Bay and P. A. Newman, Radiation Research 14, No. 5, 566-572 (May 1961).

A century-old razor, S. J. Rosenberg, Metal Progress, 94-96 (1961).

Some applications of Hardy's formula in Brownian motion (Abstract), A. Chaffari, Am. Math. Soc. Notices 8, No. 54, 238 (June 1961).

On the classical approximation in the statistical theory of mass spectra, H. M. Rosenstock, J. Chem. Phys. 34, 2182 (1961).

Optical calibration of vibration pickups at small amplitudes, V. A. Schmidt, S. Edelman, E. R. Smith, and E. Jones, J. Acoust. Soc. Am. 33, Mo. 6, 748-751 (June 1961).

Superconductivity of $\mathrm{Nb}_{3} \mathrm{Sn}$ in pulsed fields of 185 kilogauss, V. D. Arp, R. H. Kropschot, and J. H. Wilson, Phys. Rev. Letters 6, No. 9, 542-453 (May 1961).

Proposed nomenclature for linear viscoslastic behavior, $H$. Leaderman and F. Schwarzl, Rheologica Acta 1, No. 4/6, 674-676 (1961).

FM and SSB radiotelephone tests on a VHF ionospheric scatter link during multipath conditions, IRE Trans. Commun. Systems CS-8, No. 3, 183-186 (Sept. 1960).

*Publications for which a price is indicated (except for Technical Notes) are available only from the Superintendent of Documents, U.S. Government Printing Office, Washington 25, D.C. (foreign postage, one-fourth additional). Technical Notes are available only from the Office of Technical Services, U.S. Department of Commerce, Washington 25, D.C. (Order by PB number.) Reprints from outside journals and the NBS Journal of Research may often be obtained directly from the authors. 